\title{
FRAMED MOTIVES OF RELATIVE MOTIVIC SPHERES
}

\author{
GRIGORY GARKUSHA, ALEXANDER NESHITOV, AND IVAN PANIN
}

\begin{abstract}
The category of framed correspondences $\mathrm{Fr}_{*}(k)$ and framed sheaves were invented by Voevodsky in his unpublished notes [Voe]. Based on the theory, framed motives are introduced and studied in [GP1]. These are Nisnivich sheaves of $S^{1}$-spectra and the major computational tool of [GP1]. The aim of this paper is to show the following result which is essential in proving the main theorem of [GP1]: given an infinite perfect base field $k$, any $k$-smooth scheme $X$ and any $n \geqslant 1$, the map of simplicial pointed Nisnevich sheaves $\left(-, \mathbb{A}^{1} / / \mathbb{G}_{m}\right)_{+}^{\wedge n} \rightarrow T^{n}$ induces a Nisnevich local level weak equivalence of $S^{1}$-spectra

$$
M_{f r}\left(X \times\left(\mathbb{A}^{1} / / \mathbb{G}_{m}\right)^{\wedge n}\right) \rightarrow M_{f r}\left(X \times T^{n}\right) .
$$
\end{abstract}

Moreover, it is proven that the sequence of $S^{1}$-spectra

$$
M_{f r}\left(X \times T^{n} \times \mathbb{G}_{m}\right) \rightarrow M_{f r}\left(X \times T^{n} \times \mathbb{A}^{1}\right) \rightarrow M_{f r}\left(X \times T^{n+1}\right)
$$

is locally a homotopy cofiber sequence in the Nisnevich topology. Another important result of this paper shows that homology of framed motives is computed as linear framed motives in the sense of [GP1]. This computation is crucial for the whole machinery of framed motives [GP1].

\section{IntRODUCTION}

Based on Voevodsky's theory of framed correspondences [Voe], the machinery of framed motives has been introduced and developed in [GP1]. This machinery leads to serious computations not only in motivic homotopy theory, but also in classical algebraic topology. One of such computations [GP1, Theorem 11.9] states that if $k$ is the field of complex numbers, then the framed motive $M_{f r}(\mathrm{pt})(\mathrm{pt})$ of the point $\mathrm{pt}=\operatorname{Spec}(k)$ evaluated at pt has the stable homotopy type of the classical sphere spectrum $\Sigma_{S^{1}}^{\infty}\left(S^{0}\right)$. In particular, the sphere spectrum $\Sigma_{S^{1}}^{\infty}\left(S^{0}\right)$ can be computed in terms of algebraic varieties only.

The key ingredients for this computation are the theorem [GP1, Theorem 11.1] computing the motivic sphere spectrum in terms of twisted framed motives, the Cancellation Theorem for framed motives [AGP] as well as a result by Levine [L, Corollary 2]. In turn, a key ingredient for proving [GP1, Theorem 11.1] is this: for each integer $n \geqslant 1$ the canonical morphism of motivic spaces $C_{*} \operatorname{Fr}\left(\left(\mathbb{A}^{1} / / \mathbb{G}_{m}\right)^{\wedge n}\right) \rightarrow$ $C_{*} \operatorname{Fr}\left(T^{n}\right)$ is a Nisnevich local equivalence. But this is exactly a partial case of the first statement of Theorem 1.1 proven in this paper.

2020 Mathematics Subject Classification. Primary 14F42; Secondary 55P42.

Key words and phrases. Motivic homotopy theory, framed motives, motivic spheres.

This paper was partly written during the visit of the second author to Swansea University. He would like to thank the University for its kind hospitality.

The third author was supported by a grant from the Government of the Russian Federation (agreement 075-15-2019-1620). 
The machinery of framed motives also produces explicit fibrant resolutions for the suspension $\mathbb{P}^{1}$-spectrum $\Sigma_{\mathbb{P}^{1}}^{\infty} X_{+}$of a $k$-smooth algebraic variety $X \in S m / k[\mathrm{GP} 1$, Theorem 4.1] or, more generally, for the suspension $\mathbb{P}^{1}$-spectrum $\Sigma_{\mathbb{P} 1}^{\infty} \mathcal{X}$ of any pointed motivic space $\mathcal{X}$ [GP1, Section 10]. Theorem 1.1 and its Corollary 8.1 proven in this paper play a key role in the proof of [GP1, Theorem 4.1] as well as for results of [GP1, Section 10].

Indeed, one of the major steps in the proof of [GP1, Theorem 4.1] is to show that for each $n \geqslant 1$ the canonical morphism $C_{*} \operatorname{Fr}\left(X \times T^{n}\right)_{f} \rightarrow \underline{\operatorname{Hom}}\left(\mathbb{P}^{\wedge 1}, C_{*} \operatorname{Fr}(X \times\right.$ $\left.T^{n+1}\right)_{f}$ ) is a sectionwise weak equivalence of motivic spaces (see [GP1, Section 9] for details). Here $f$ stands for a fibrant replacement within the injective local model structure. Theorem 1.1 and its Corollary 8.1 proven in this paper are used in [GP1, Section 9] to reduce this major step to the following two statements. The first one requires to show that for each $Y \in S m / k$ the canonical morphism

$$
M_{f r}(Y)_{\mathrm{f}} \rightarrow \underline{\operatorname{Hom}}\left(\mathbb{G}_{m}^{\wedge 1}, M_{f r}\left(Y \times \mathbb{G}_{m}^{\wedge 1}\right)_{\mathrm{f}}\right)
$$

is a sectionwise stable equivalence of $S^{1}$-spectra. Here "f" refers to a stable Nisnevich local fibrant replacement of $S^{1}$-spectra. The second one requires to show that for each $Z \in S m / k$ the canonical morphism

$$
M_{f r}(Z) \rightarrow \underline{\operatorname{Hom}}\left(S^{1}, M_{f r}\left(Z \otimes S^{1}\right)\right)
$$

is a sectionwise stable equivalence of $S^{1}$-spectra. As explained in [GP1, Section 9] the first statement is nothing but the Cancellation Theorem for framed motives [AGP, Theorem A]. The second statement follows from the Additivity Theorem in [GP1, Theorem 6.5].

In order to formulate Theorem 1.1 below, let us briefly describe the relevant notions. Let $\operatorname{Fr}_{0}(k)$ be the category whose objects are those of $S m / k$ and whose morphism set between $X$ and $Y$ is given by the set of framed correspondences of level zero [Voe, Example 2.1], [GP1, Definition 2.1]. As it is shown in [GP1, Section 5], the category of framed correspondences of level zero $\operatorname{Fr}_{0}(k)$ has an action by finite pointed sets $U \otimes K:=\bigsqcup_{K \backslash *} U$ with $U \in S m / k$ and $K$ a finite pointed set. The cone of $U$ is the simplicial object $U \otimes I$ in $\operatorname{Fr}_{0}(k)$, where $(I, 1)$ is the pointed simplicial set $\Delta[1]$ with basepoint 1 . There is a natural morphism $i_{0}: U \rightarrow U \otimes I$ in $\Delta^{\mathrm{op}} \operatorname{Fr}_{0}(k)$. Given an inclusion of smooth schemes $j: U \hookrightarrow Y$, denote by $Y / / U$ a simplicial object in $\operatorname{Fr}_{0}(k)$ which is obtained by taking the pushout of the diagram $Y \hookleftarrow U \stackrel{i_{0}}{\hookrightarrow} U \otimes I$ in $\Delta^{\mathrm{op}} \operatorname{Fr}_{0}(k)$. The simplicial object $Y / / U$ termwise equals $Y, Y \sqcup U, Y \sqcup U \sqcup U, \ldots$. Recall that the category $\operatorname{Fr}_{0}(k)$ is a full subcategory of $\operatorname{SmOp}\left(\mathrm{Fr}_{0}(k)\right)$ (see [GP1, Section 5] for definitions). If $j: U \hookrightarrow Y$ is an open inclusion, then we have an object $(Y, U) \in S m O p\left(\operatorname{Fr}_{0}(k)\right)$. Regard it as an object of the category $\Delta^{\mathrm{op}}\left(\operatorname{SmOp}\left(\mathrm{Fr}_{0}(k)\right)\right)$ which is constant in the simplicial direction. Then there is an obvious morphism $\alpha: Y / / U \rightarrow(Y, U)$ in the latter category. Namely, at each simplicial level $m, \alpha$ is a morphism in $\operatorname{SmOp}\left(\operatorname{Fr}_{0}(k)\right)$

$$
\alpha_{m}: Y \sqcup U \sqcup \ldots \sqcup U \rightarrow(Y, U)
$$

such that $\left.\alpha_{m}\right|_{Y}=\operatorname{id}_{Y}:(Y, \emptyset) \rightarrow(Y, U),\left.\alpha_{m}\right|_{U}=j:(U, \emptyset) \rightarrow(Y, U)$. The categories $\operatorname{Fr}_{0}(k)$ and $\operatorname{SmOp}\left(\mathrm{Fr}_{0}(k)\right)$ are both symmetric monoidal (see [GP1, Section 5]) and the inclusion of $\operatorname{Fr}_{0}(k)$ into $\operatorname{SmOp}\left(\mathrm{Fr}_{0}(k)\right)$ is a strict monoidal functor. Thus we have the $n$th monoidal power $(Y / / U)^{\wedge n}$ (respectively $\left.(Y, U)^{\wedge n}\right)$ of $Y / / U$ (respectively of $(Y, U)$ ) and a morphism $\alpha^{\wedge n}:(Y / / U)^{\wedge n} \rightarrow(Y, U)^{\wedge n}$, which is the $n$th monoidal power of $\alpha$. For any $k$-smooth variety $X$ one has the morphism 
$\operatorname{id}_{X} \times \alpha^{\wedge n}: X \times(Y / / U)^{\wedge n} \rightarrow X \times(Y, U)^{\wedge n}$ in $\Delta^{\mathrm{op}}\left(S m O p\left(\operatorname{Fr}_{0}(k)\right)\right)$. Applying the functor $M_{f r}$ from [GP1, Definition 5.2] to this morphism, we get a morphism of framed $S^{1}$-spectra

$$
M_{f_{r}}\left(X \times(Y / / U)^{\wedge n}\right) \stackrel{M_{f r}\left(\mathrm{id}_{X} \times \alpha^{\wedge n}\right)}{\longrightarrow} M_{f r}\left(X \times(Y, U)^{\wedge n}\right) .
$$

In the special case when $(Y, U)=\left(\mathbb{A}^{1}, \mathbb{G}_{m}\right)$, the $S^{1}$-spectrum $M_{f r}\left(X \times(Y, U)^{\wedge n}\right)$ will be denoted by $M_{f r}\left(X \times T^{n}\right)$. Note that $X \times\left(\mathbb{A}^{1}, \mathbb{G}_{m}\right)^{\wedge n}=\left(X \times \mathbb{A}^{n}, X \times\left(\mathbb{A}^{n}-\right.\right.$ $0)$ ).

As explained above, the computation of an explicit motivically fibrant resolution of the suspension $\mathbb{P}^{1}$-spectrum $\Sigma_{\mathbb{P}^{1}}^{\infty} X_{+}$, given in [GP1, 4.1], requires Theorem 1.1 and its Corollary 8.1.

Theorem 1.1. Let $k$ be an infinite perfect field. For any $k$-smooth scheme $X \in$ $S m / k$ and any $n \geqslant 1$, the morphism $\alpha^{\wedge n}:\left(\mathbb{A}^{1} / / \mathbb{G}_{m}\right)^{\wedge n} \rightarrow\left(\mathbb{A}^{1}, \mathbb{G}_{m}\right)^{\wedge n}$ of simplicial objects in $\operatorname{SmOp}\left(\mathrm{Fr}_{0}(k)\right)$ induces a level Nisnevich local weak equivalence of $S^{1}$ spectra

$$
M_{f r}\left(\operatorname{id}_{X} \times \alpha^{\wedge n}\right): M_{f r}\left(X \times\left(\mathbb{A}^{1} / / \mathbb{G}_{m}\right)^{\wedge n}\right) \rightarrow M_{f r}\left(X \times T^{n}\right) .
$$

Moreover, the sequence of $S^{1}$-spectra

$$
M_{f r}\left(X \times T^{n} \times \mathbb{G}_{m}\right) \rightarrow M_{f r}\left(X \times T^{n} \times \mathbb{A}^{1}\right) \rightarrow M_{f r}\left(X \times T^{n+1}\right)
$$

is locally a homotopy cofiber sequence in the Nisnevich topology.

The main goal of the paper is to prove this theorem and its consequence concerning the natural morphism (1.1) described below. The first part of Theorem 1.1 states that the $S^{1}$-spectum $M_{f r}\left(X \times T^{n}\right)$ of the relative motivic sphere $X \times \mathbb{A}^{n} /(X \times$ $\left.\left(\mathbb{A}^{n}-0\right)\right)$ can locally be computed as the $S^{1}$-spectrum $M_{f r}\left(X \times\left(\mathbb{A}^{1} / / \mathbb{G}_{m}\right)^{\wedge n}\right)$ of the simplicial object $X \times\left(\mathbb{A}^{1} / / \mathbb{G}_{m}\right)^{\wedge n}$ in $\operatorname{Fr}_{0}(k)$. Another consequence of the theorem says that for every $n \geqslant 0$ the natural morphism

$$
M_{f r}\left(X \times T^{n} \times\left(\mathbb{A}^{1} / / \mathbb{G}_{m}\right)\right) \rightarrow M_{f r}\left(X \times T^{n+1}\right)
$$

is a level weak equivalence of $S^{1}$-spectra locally in the Nisnevich topology (see Corollary 8.1). As explained above, the proof of $[\mathrm{GP} 1,4.1]$ depends on the local equivalence (1.1).

Theorem 1.1 and its Corollary 8.1 concerning the local equivalence (1.1) are proved in Section 8.

We have already discussed the importance of Theorem 1.1. Let us explain why it is highly non-trivial. Indeed, using Linearisation Theorem 1.2 stated below one can check that the second assertion of Theorem 1.1 is equivalent to the assertion that the morphism $\tau$ from Theorem 1.3 is a Nisnevich local equivalence. For simplicity take $X=\mathrm{pt}$ and $n=0$. In this case the domain of $\tau$ is the complex $C_{*}\left(\mathbb{Z F}\left(\mathbb{A}^{1}\right) / \mathbb{Z F}\left(\mathbb{G}_{m}\right)\right)$ of linear framed presheaves. The codomain of $\tau$ is the complex $C_{*} \mathbb{Z F}(T)$ of linear framed presheaves and $\tau=C_{*}(p)$, where

$$
p: \mathbb{Z F}\left(\mathbb{A}^{1}\right) / \mathbb{Z} \mathrm{F}\left(\mathbb{G}_{m}\right) \rightarrow \mathbb{Z} \mathrm{F}(T)
$$

is a natural morphism of presheaves. The morphism $p$ is far from being Nisnevich locally an isomorphism, because otherwise Lemma 5.4 would imply that $\tau=C_{*}(p)$ is locally a quasi-isomorphism of complexes. However, it is not the case: the morphism of presheaves

$$
p: \mathbb{Z F}\left(\mathbb{A}^{1}\right) / \mathbb{Z F}\left(\mathbb{G}_{m}\right) \rightarrow \mathbb{Z F}(T)
$$


is not locally an isomorphism (see Example 3.3). This morphism is locally a monomorphism only. Its image is locally $\mathbb{Z} \mathrm{F}^{q f}(T)$ with $\mathbb{Z} \mathrm{F}^{q f}(T)$ being a certain linear framed subpresheaf of $\mathbb{Z F}(T)$. The presheaf $\mathbb{Z F}^{q f}(T)$ is of independent interest. An advantage of $\mathbb{Z F}^{q f}(T)$ is that its sections are described by explicit geometric data. Using properties of $\mathbb{Z F}^{q f}(T)$, Theorem 1.3 then splits in two further Theorems 1.4 and 1.5 formulated below. As we can see, Theorem 1.1 requires several non-trivial reductions showing that the theorem itself is a highly non-trivial result.

In the rest of the introduction we describe the steps required for the proof of Theorem 1.1 as well as fix some notation. The following theorem (see Theorem 1.2) is crucial in the analysis of framed motives. It allows to reduce many computations for framed motives of algebraic varieties to analogous computations for complexes of linear framed presheaves, which are normally much simpler. In particular, the theorem computes homology of framed motives. As an application of Theorem 1.2, Theorem 1.1 reduces to Theorem 1.3. It is as well worthwhile to mention another similar application of this kind. In [AGP] the Cancellation Theorem for framed motives of algebraic varieties is proved by reducing it to complexes of linear framed presheaves.

We are now in a position to formulate the Linearisation Theorem.

Theorem 1.2 (Linearisation). For any integer $m \geqslant 0$, the natural morphism of framed $S^{1}$-spectra

$$
\lambda_{X \times T^{m}}: \mathbb{Z} \mathrm{Fr}_{*}^{S^{1}}\left(X \times T^{m}\right) \rightarrow E M\left(\mathbb{Z F}_{*}\left(-, X \times T^{m}\right)\right)
$$

is a schemewise stable equivalence. Moreover, the natural morphism of framed $S^{1}$ spectra

$$
l_{X \times T^{m}}: \mathbb{Z} M_{f r}\left(X \times T^{m}\right) \rightarrow L M_{f r}\left(X \times T^{m}\right)
$$

is a schemewise stable equivalence. In particular, for any $U \in S m / k$ one has

$$
\pi_{*}\left(\mathbb{Z} M_{f r}\left(X \times T^{m}\right)(U)\right)=H_{*}\left(\mathbb{Z F}\left(\Delta \bullet \times U, X \times T^{m}\right)\right)=H_{*}\left(C_{*} \mathbb{Z} \mathrm{F}\left(U, X \times T^{m}\right)\right) .
$$

Notation used in Theorem 1.2 is explained in Section 8. The theorem itself is proved in Appendix B. Thus in order to prove Theorem 1.1, it is sufficient to prove the following theorem.

Theorem 1.3. Let $k$ be an infinite perfect field. For any $k$-smooth scheme $X$ and any $n \geqslant 0$ the natural map of complexes of linear framed presheaves

$$
\tau: C_{*} \mathbb{Z F}\left(X \times T^{n} \times \mathbb{A}^{1}\right) / C_{*} \mathbb{Z} \mathrm{F}\left(X \times T^{n} \times \mathbb{G}_{m}\right) \rightarrow C_{*} \mathbb{Z F}\left(X \times T^{n+1}\right)
$$

is a Nisnevich local equivalence.

The proof of Theorem 1.3 splits in two steps, each of which is of independent interest. Firstly we introduce a linear framed subpresheaf $\mathbb{Z F}^{q f}\left(X \times T^{n+1}\right)$ of the linear framed presheaf $\mathbb{Z} \mathrm{F}\left(X \times T^{n+1}\right)$ and prove the following

Theorem 1.4. For any $k$-smooth scheme $X$ and any $n \geqslant 0$, the natural morphism

$$
\mathbb{Z} \mathrm{F}\left(X \times T^{n} \times \mathbb{A}^{1}\right) / \mathbb{Z} \mathrm{F}\left(X \times T^{n} \times \mathbb{G}_{m}\right) \rightarrow \mathbb{Z F}^{q f}\left(X \times T^{n+1}\right)
$$

of linear framed presheaves is an isomorphism locally in the Nisnevich topology.

By applying the Suslin complex, one can show that the natural morphism of complexes of linear framed presheaves

$$
\mu: C_{*} \mathbb{Z F}\left(X \times T^{n} \times \mathbb{A}^{1}\right) / C_{*} \mathbb{Z F}\left(X \times T^{n} \times \mathbb{G}_{m}\right) \rightarrow C_{*} \mathbb{Z F}^{q f}\left(X \times T^{n+1}\right)
$$


is locally a quasi-isomorphism in the Nisnevich topology (see Proposition 5.5 for details).

Secondly using a moving lemma discussed in Section 7, we then prove the following

Theorem 1.5. The inclusion of complexes of linear framed presheaves

$$
C_{*} \mathbb{Z F}^{q f}\left(X \times T^{n+1}\right) \hookrightarrow C_{*} \mathbb{Z F}\left(X \times T^{n+1}\right)
$$

is locally a quasi-isomorphism in the Zariski topology.

Clearly, Theorem 1.5 together with the Nisnevich local quasi-isomorphism (1.2) imply Theorem 1.3.

Throughout the paper we denote by $S m / k$ the category of smooth separated schemes of finite type over the base field $k$. The subcategory of affine smooth $k$-varieties is denoted by $\operatorname{AffSm} / k$. Given a scheme $W$ and a family of regular function $\varphi_{1}, \ldots, \varphi_{m}$ on $W$, we write $Z\left(\varphi_{1}, \ldots, \varphi_{m}\right)$ to denote the closed subset in $W$ which is the common vanishing locus of the family $\varphi_{1}, \ldots, \varphi_{m}$. Whenever we speak about $\Gamma$-spaces we follow the terminology of Bousfield-Friedlander [BF]. The category of pointed Nisnevich sheaves (respectively framed sheaves) will be denoted by $S h v_{\bullet}(S m / k)\left(\right.$ respectively $\left.S h v_{\bullet}^{f r}(S m / k)\right)$. We shall also write $\operatorname{Pre}_{\mathcal{A} b}(S m / k)$ to denote the category of presheaves of Abelian groups on $S m / k$. Whenever the authors write "locally", it is always assumed "locally in the Nisnevich topology". They also stress that motivic equivalences are not used anywhere in this paper except the proof of Lemma 5.4.

\section{Framed presheaves $\operatorname{Fr}(-, Y /(Y-S))$ And $\mathbb{Z F}(-, Y /(Y-S))$}

Definition 2.1. (I) Let $Y$ be a $k$-smooth scheme and $S \subset Y$ be a closed subset and let $U \in S m / k$. An explicit framed correspondence of level $m \geqslant 0$ from $U$ to $Y /(Y-S)$ consists of the following tuples:

$$
\left(Z, W, \varphi_{1}, \ldots, \varphi_{m} ; g: W \rightarrow Y\right),
$$

where $Z$ is a closed subset of $U \times \mathbb{A}^{m}$, finite over $U, W$ is an étale neighborhood of $Z$ in $U \times \mathbb{A}^{m}, \varphi_{1}, \ldots, \varphi_{m}$ are regular functions on $W, g$ is a regular map such that $Z=Z\left(\varphi_{1}, \ldots, \varphi_{m}\right) \cap g^{-1}(S)$. The set $Z$ is called the support of the explicit framed correspondence. We shall also write quadruples $\Phi=(Z, W, \varphi ; g)$ to denote explicit framed correspondences.

(II) Two explicit framed correspondences $(Z, W, \varphi ; g)$ and $\left(Z^{\prime}, W^{\prime}, \varphi^{\prime} ; g^{\prime}\right)$ of level $m$ are said to be equivalent if $Z=Z^{\prime}$ and there exists an étale neighborhood $W^{\prime \prime}$ of $Z$ in $W \times_{\mathbb{A}_{U}^{m}} W^{\prime}$ such that $\varphi \circ p r$ agrees with $\varphi^{\prime} \circ p r^{\prime}$ and the morphism $g \circ p r$ agrees with $g^{\prime} \circ p r^{\prime}$ on $W^{\prime \prime}$.

(III) A framed correspondence of level $m$ from $U$ to $Y /(Y-S)$ is the equivalence class of an explicit framed correspondence of level $m$ from $U$ to $Y /(Y-S)$. We write $\operatorname{Fr}_{m}(U, Y /(Y-S))$ to denote the set of framed correspondences of level $m$ from $U$ to $Y /(Y-S)$. We regard it as a pointed set whose distinguished point is the class $0_{Y /(Y-S), m}$ of the explicit correspondence $(Z, W, \varphi ; g)$ with $W=\emptyset$.

(IV) If $S=Y$ then the set $\operatorname{Fr}_{m}(U, Y /(Y-S))$ is denoted by $\operatorname{Fr}_{m}(U, Y)$ and is called the set of framed correspondences of level $m$ from $U$ to $Y$.

(V) Following Voevodsky [Voe], the category of framed correspondences $\mathrm{Fr}_{*}(k)$ has objects those of $S m / k$ and its morphisms are given by the sets $\operatorname{Fr}_{*}(U, Y):=$ 
$\bigsqcup_{m \geqslant 0} \operatorname{Fr}_{m}(U, Y), U, Y \in S m / k$. The subcategory of $\operatorname{Fr}_{*}(k)$ of framed correspondences of level zero will be denoted by $\operatorname{Fr}_{0}(k)$.

(VI) A framed presheaf is just a contravariant functor from the category $\operatorname{Fr}_{*}(k)$ to sets.

Let $X, Y$ and $S$ be $k$-smooth schemes and let

$$
\Psi=\left(Z^{\prime}, \mathbb{A}^{k} \times V \stackrel{\left(\alpha, \pi^{\prime}\right)}{\longleftarrow} W^{\prime}, \psi_{1}, \psi_{2}, \ldots, \psi_{k} ; g: W^{\prime} \rightarrow U\right) \in \operatorname{Fr}_{k}(V, U)
$$

be an explicit correspondence of level $k$ from $V$ to $U$ and let

$$
\Phi=\left(Z, \mathbb{A}^{m} \times U \stackrel{(\beta, \pi)}{\longleftarrow} W, \varphi_{1}, \varphi_{2}, \ldots, \varphi_{m} ; g^{\prime}: W \rightarrow Y\right) \in \operatorname{Fr}_{m}(U, Y /(Y-S))
$$

be an explicit correspondence of level $m$ from $U$ to $Y /(Y-S)$. We define $\Psi^{*}(\Phi)$ as an explicit correspondence of level $k+m$ from $V$ to $Y /(Y-S)$ as

$$
\begin{array}{r}
\left(Z \times_{U} Z^{\prime}, \mathbb{A}^{k+m} \times V \stackrel{\left(\alpha, \beta, \pi^{\prime}\right)}{\longleftarrow} W^{\prime} \times_{U} W, \psi_{1}, \psi_{2}, \ldots, \psi_{k}, \varphi_{1}, \varphi_{2}, \ldots, \varphi_{m},, g^{\prime} \circ p r_{W}\right) \\
\in \operatorname{Fr}_{k+m}(V, Y /(Y-S)) .
\end{array}
$$

Clearly, the pull-back operation $(\Psi, \Phi) \mapsto \Psi^{*}(\Phi)$ of explicit correspondences respects the equivalence relation on them. We get a pairing

$$
\operatorname{Fr}_{k}(V, U) \times \operatorname{Fr}_{m}(U, Y /(Y-S)) \rightarrow \operatorname{Fr}_{k+m}(V, Y /(Y-S))
$$

making $\operatorname{Fr}_{*}(U, Y /(Y-S)):=\bigsqcup_{m \geqslant 0} \operatorname{Fr}_{m}(-, Y /(Y-S))$ a $\operatorname{Fr}_{*}(k)$-presheaf.

Let $X, Y, S$ and $T$ be smooth schemes. There is an external product

$$
\operatorname{Fr}_{m}(U, Y /(Y-S)) \times \operatorname{Fr}_{n}(\mathrm{pt}, \mathrm{pt}) \stackrel{-\otimes-}{\longrightarrow} \operatorname{Fr}_{m+n}(U, Y /(Y-S))
$$

given by

$$
\begin{aligned}
\left(\left(Z, W, \varphi_{1}, \varphi_{2}, \ldots, \varphi_{m} ; g\right),\left(Z^{\prime}, W^{\prime}, \psi_{1}, \ldots, \psi_{n}\right)\right) & \\
& \mapsto\left(Z \times Z^{\prime}, W \times W^{\prime}, \varphi_{1}, \varphi_{2}, \ldots, \varphi_{m}, \psi_{1}, \ldots, \psi_{n} ; g\right) .
\end{aligned}
$$

Set $\sigma:=\left(\{0\}, \mathbb{A}^{1}, i d: A^{1} \rightarrow \mathbb{A}^{1}\right.$, const $\left.: \mathbb{A}^{1} \rightarrow p t\right) \in \operatorname{Fr}_{1}(p t, p t)$. Denote by

$$
\Sigma: \operatorname{Fr}_{m}(U, Y /(Y-S)) \rightarrow \operatorname{Fr}_{m+1}(U, Y /(Y-S))
$$

the map $\Phi \mapsto \Phi \otimes \sigma$. Following Voevodsky [Voe] we give the following

Definition 2.2. We shall refer to the set

$$
\begin{aligned}
\operatorname{Fr}(U, Y /(Y-S)):=\operatorname{colim}\left(\operatorname{Fr}_{0}(U, Y /(Y-S)) \stackrel{\Sigma}{\rightarrow}\right. & \operatorname{Fr}_{1}(U, Y /(Y-S)) \\
& \left.\stackrel{\Sigma}{\longrightarrow} \operatorname{Fr}_{2}(U, Y /(Y-S)) \ldots\right)
\end{aligned}
$$

as the set stable framed correspondences from $U$ to $Y /(Y-S)$. Clearly, $\operatorname{Fr}(-, Y /(Y-$ $S)$ ) is a framed presheaf of pointed sets with the empty framed correspondence being the distinguished point.

Clearly, $\operatorname{Fr}(-, Y /(Y-S))$ is even a framed functor in the sense of Voevodsky [Voe] meaning that $\operatorname{Fr}(\emptyset)=*$ and $\operatorname{Fr}\left(U_{1} \sqcup U_{2}, Y /(Y-S)\right)=\operatorname{Fr}\left(U_{1}, Y /(Y-S)\right) \times$ $\operatorname{Fr}\left(U_{2}, Y /(Y-S)\right)$.

Definition 2.3 (cf. [GP1]). Let $Y \in S m / k$ and $S \subset Y$ be as in Definition 2.1. Let $U$ be a $k$-smooth scheme. Denote by

$\diamond \mathbb{Z} \operatorname{Fr}_{m}(U, Y /(Y-S)):=\widetilde{\mathbb{Z}}\left[\operatorname{Fr}_{m}(U, Y /(Y-S))\right]=\mathbb{Z}\left[\operatorname{Fr}_{m}(U, Y /(Y-S))\right] / \mathbb{Z} \cdot$ $0_{Y /(Y-S), m}$, i.e the free abelian group generated by the set $\operatorname{Fr}_{m}(U, Y /(Y-$ $S)$ ) modulo $\mathbb{Z} \cdot 0_{Y /(Y-S), m}$ 
$\diamond \mathbb{Z F}_{m}(U, Y /(Y-S)):=\mathbb{Z F r}_{m}(U, Y /(Y-S)) / A$, where $A$ is the subgroup generated by the elements

$$
\begin{aligned}
\left(Z \sqcup Z^{\prime}, W,\left(\varphi_{1}, \varphi_{2}, \ldots, \varphi_{m}\right) ; g\right)- & \left(Z, W \backslash Z^{\prime},\left.\left(\varphi_{1}, \varphi_{2}, \ldots, \varphi_{m}\right)\right|_{W \backslash Z^{\prime}} ;\left.g\right|_{W \backslash Z^{\prime}}\right)- \\
& -\left(Z^{\prime}, W \backslash Z,\left.\left(\varphi_{1}, \varphi_{2}, \ldots, \varphi_{m}\right)\right|_{W \backslash Z} ;\left.g\right|_{W \backslash Z}\right) .
\end{aligned}
$$

The elements of $\mathbb{Z F}_{m}(U, Y /(Y-S))$ are called linear framed correspondences from $U$ to $Y /(Y-S)$ of level $m$.

Definition 2.4. Define $\mathrm{F}_{m}(U, Y /(Y-S)) \subset \operatorname{Fr}_{m}(U, Y /(Y-S))$ as the subset consisting of $(Z, W, \varphi ; g) \in \operatorname{Fr}_{m}(U, Y /(Y-S))$ such that $Z$ is connected.

Clearly, the set $\mathrm{F}_{m}(U, Y /(Y-S))-0_{m}$ is a free basis of the abelian group $\mathbb{Z F}_{m}(U, Y /(Y-S))$. However, the assignment $U \mapsto \mathrm{F}_{m}(U, Y /(Y-S))$ is not a presheaf even on the category $S m / k$.

Indeed, if $u \stackrel{i}{\rightarrow} U$ is a closed point and $(Z, W, \varphi ; g) \in \mathrm{F}_{m}(U, Y /(Y-S)) \subseteq$ $\operatorname{Fr}_{m}(U, Y /(Y-S))$, then the support of $i^{*}(Z, W, \varphi ; g) \in \operatorname{Fr}_{m}(u, Y /(Y-S))$ is the closed subsetset $Z_{u}$ in $\mathbb{A}_{u}^{m}$. Clearly, $Z_{u}$ is not connected in general.

The category of linear framed correspondences $\mathbb{Z} \mathrm{F}_{*}(k)$ is defined in [GP1]. We shall also refer to contravariant functors from the category $\mathbb{Z} \mathrm{F}_{*}(k)$ to Abelian groups as linear framed presheaves.

Set $\mathbb{Z} \mathrm{F}_{*}(U, Y /(Y-S))=\bigoplus_{m \geqslant 0} \mathbb{Z F}_{m}(U, Y /(Y-S))$. The pairing (2.1) induces in a natural way a bilinear pairing

$$
\mathbb{Z F}_{k}(V, U) \times \mathbb{Z F}_{m}(U, Y /(Y-S)) \rightarrow \mathbb{Z F}_{k+m}(U, Y /(Y-S)) .
$$

The latter pairing makes $\mathbb{Z F}_{*}(-, Y /(Y-S))$ a linear framed presheaf.

The external product (2.2) induces in a natural way an external product of the form

$$
\left.\mathbb{Z} \mathrm{F}_{m}(U, Y /(Y-S))\right) \times \mathbb{Z}_{n}(\mathrm{pt}, \mathrm{pt}) \stackrel{-\bigotimes-}{\longrightarrow} \mathbb{Z}_{m+n}(U, Y /(Y-S))
$$

Let $Y \in S m / k$ and $S \subset Y$ be as in Definition 2.1. One of the main linear framed presheaves of this paper we are interested in is defined as

$$
\begin{aligned}
\mathbb{Z} \mathrm{F}(-, Y /(Y-S))=\operatorname{colim}\left(\mathbb{Z F}_{0}(-, Y /(Y-S))\right. & \stackrel{\Sigma}{\rightarrow} \mathbb{Z}_{1}(-, Y /(Y-S)) \\
& \left.\stackrel{\Sigma}{\rightarrow} \mathbb{Z}_{2}(-, Y /(Y-S)) \stackrel{\Sigma}{\rightarrow} \ldots\right) .
\end{aligned}
$$

Definition 2.5. Following [GP1, Definition 5.1], define a category $S m O p\left(\operatorname{Fr}_{0}(k)\right)$, which will often be used in our constructions. Its objects are pairs $(X, U)$, where $X \in S m / k$ and $U \subset X$ is an open subset. A morphism between $(X, U)$ and $\left(X^{\prime}, U^{\prime}\right)$ in $\operatorname{SmOp}\left(\operatorname{Fr}_{0}(k)\right)$ is a morphism $f \in \operatorname{Fr}_{0}\left(X, X^{\prime}\right)$ such that $f(U) \subset U^{\prime}$. We shall also identify $X \in S m / k$ with the pair $(X, \emptyset) \in S m O p\left(\operatorname{Fr}_{0}(k)\right)$.

The category $S m O p\left(\operatorname{Fr}_{0}(k)\right)$ is symmetric monoidal with the monoidal product $\wedge$ given by

$$
(X, U) \wedge(Y, V):=(X \times Y, X \times V \cup U \times Y) .
$$

The point pt is its monoidal unit. If $U=X-S$ and $V=Y-T$ then

$(X, X-S) \wedge(Y, Y-T)=(X \times Y, X \times(Y-T) \cup(X-S) \times Y)=(X \times Y, X \times Y-S \times T)$.

If $X \in S m / k$ we will also write $X \times(Y, V)$ (respectively, $(Y, V) \times X)$ to denote the object $(X, \emptyset) \wedge(Y, V)=(X \times Y, X \times V)$ (respectively, $(Y, V) \wedge(X, \emptyset)=(Y \times X, V \times$ $X))$ of $S m O p\left(\operatorname{Fr}_{0}(k)\right)$. Also, by $(X, U) \sqcup(Y, V)$ we shall mean $(X \sqcup Y, U \sqcup V)$. 
Define now covariant functors $\operatorname{Fr}_{m}: S m O p\left(\operatorname{Fr}_{0}(k)\right) \rightarrow S h v_{\bullet}(S m / k)$ and $\mathrm{Fr}:$ $\operatorname{SmOp}\left(\operatorname{Fr}_{0}(k)\right) \rightarrow S h v_{\bullet}^{f r}(S m / k)$.

Construction 2.6 (The covariant functor $\left.\operatorname{Fr}_{m}\right)$. For any object $(Y, Y-S)$ in $S m O p\left(\operatorname{Fr}_{0}(k)\right)$ and any integer $m \geqslant 0$, the value of $\operatorname{Fr}_{m}$ at $(Y, Y-S)$ is the sheaf $\operatorname{Fr}_{m}(-, Y /(Y-S))$. For each $\mathrm{f} \in \operatorname{Hom}_{S m O p\left(\operatorname{Fr}_{0}(k)\right)}\left((Y, Y-S),\left(Y^{\prime}, Y^{\prime}-S^{\prime}\right)\right)$, write $\mathrm{f}_{*, m}: \operatorname{Fr}_{m}(-, Y /(Y-S)) \rightarrow \operatorname{Fr}_{m}\left(-, Y^{\prime} /\left(Y^{\prime}-S^{\prime}\right)\right)$ to denote the following morphism of sheaves: given $U \in S m / k$, it takes $\left(Z, W, \varphi_{1}, \ldots, \varphi_{m} ; g\right) \in \operatorname{Fr}_{m}(U, Y /(Y-S))$ to $\left(Z\left(\varphi_{1}, \ldots, \varphi_{m}\right) \cap(\mathrm{f} \circ g)^{-1}\left(S^{\prime}\right), W, \varphi_{1}, \ldots, \varphi_{m} ; \mathrm{f} \circ g\right) \in \mathrm{Fr}_{m}\left(-, Y^{\prime} /\left(Y^{\prime}-S^{\prime}\right)\right)$. Clearly, the assignments $(Y, Y-S) \mapsto \mathrm{Fr}_{m}(-, Y /(Y-S))$ and $\mathrm{f} \mapsto \mathrm{f}_{*, m}$ form a functor

$$
\operatorname{Fr}_{m}: S m O p\left(\operatorname{Fr}_{0}(k)\right) \rightarrow S h v_{\bullet}(S m / k) .
$$

Likewise, the assignments $(Y, Y-S) \mapsto \mathbb{Z}\left[\operatorname{Fr}_{m}(-, Y /(Y-S))\right]$ and $\mathrm{f} \mapsto \mathbb{Z}\left[\mathrm{f}_{*, m}\right]$ form a functor

$$
\mathbb{Z}\left[\operatorname{Fr}_{m}\right]: S m O p\left(\operatorname{Fr}_{0}(k)\right) \rightarrow \operatorname{Pre}_{\mathcal{A} b}(S m / k)
$$

as well as the assignments $\left.(Y, Y-S) \mapsto \mathbb{Z F}_{m}(-, Y /(Y-S))\right]$ and $\mathrm{f} \mapsto \mathbb{Z}_{*, m}$ form a functor

$$
\mathbb{Z F}_{m}: \operatorname{SmOp}\left(\operatorname{Fr}_{0}(k)\right) \rightarrow \operatorname{Pre}_{\mathcal{A} b}(S m / k) .
$$

Construction 2.7 (The covariant functor Fr). For each integer $m \geqslant 0$ and each object $(Y, Y-S)$ in $S m O p\left(\operatorname{Fr}_{0}(k)\right)$, write $\Sigma_{m, Y /(Y-S)}$ for the morphism of sheaves $\Sigma: \operatorname{Fr}_{m}(-, Y /(Y-S)) \rightarrow \operatorname{Fr}_{m+1}(-, Y /(Y-S))$ given in Definition 2.2. It is easy to check that the assignment $(Y, Y-S) \mapsto \Sigma_{m, Y /(Y-S)}$ gives rise to a natural transformation of functors $\Sigma: \mathrm{Fr}_{m} \rightarrow \mathrm{Fr}_{m+1}$. Taking the colimit

$$
\operatorname{Fr}=\operatorname{colim}\left(\operatorname{Fr}_{0} \stackrel{\Sigma}{\rightarrow} \operatorname{Fr}_{1} \stackrel{\Sigma}{\rightarrow} \operatorname{Fr}_{2} \stackrel{\Sigma}{\rightarrow} \ldots\right),
$$

we get a functor from $S m O p\left(\operatorname{Fr}_{0}(k)\right)$ to $S h v_{\bullet}(S m / k)$. As mentioned in Definition 2.2 for each object $(Y, Y-S) \in S m O p\left(\operatorname{Fr}_{0}(k)\right)$ the sheaf $\operatorname{Fr}(-, Y /(Y-S))$ is naturally a framed Nisnevich sheaf. Moreover, for each morphism $f:(Y, Y-$ $S) \rightarrow\left(Y^{\prime}, Y^{\prime}-S^{\prime}\right)$ in $S m O p\left(\operatorname{Fr}_{0}(k)\right)$ the morphism $\operatorname{Fr}(f): \operatorname{Fr}(-, Y /(Y-S)) \rightarrow$ $\operatorname{Fr}\left(-, Y^{\prime} /\left(Y^{\prime}-S^{\prime}\right)\right)$ is a morphism of framed Nisnevich sheaves.

The covariant functors $(Y, Y-S) \mapsto \mathbb{Z}[\operatorname{Fr}(-, Y /(Y-S))]$ and $(Y, Y-S) \mapsto$ $\mathbb{Z} \mathrm{F}(-, Y /(Y-S))]$ from $S m O p\left(\operatorname{Fr}_{0}(k)\right)$ to $\operatorname{Pre}_{\mathcal{A} b}^{f r}(S m / k)$ are defined in a similar fashion.

Notation 2.8. Given $X \in S m / k$ and $n>0$, we will often write $\operatorname{Fr}_{m}\left(X \times T^{n}\right)$ (respectively, $\operatorname{Fr}\left(X \times T^{n}\right), \mathbb{Z}\left[\mathrm{Fr}_{m}\right]\left(X \times T^{n}\right), \mathbb{Z}[\mathrm{Fr}]\left(X \times T^{n}\right), \mathbb{Z} F_{m}\left(X \times T^{n}\right), \mathbb{Z} F(X \times$ $\left.T^{n}\right)$ ) to denote the value of the functor $\operatorname{Fr}_{m}$ (respectively, $\mathrm{Fr}, \mathbb{Z}\left[\mathrm{Fr}_{m}\right], \mathbb{Z}[\mathrm{Fr}], \mathbb{Z} F_{m}$, $\mathbb{Z} F)$ on the couple $X \times\left(\mathbb{A}^{1}, \mathbb{G}_{m}\right)^{\wedge n} \in \operatorname{SmOp}\left(\operatorname{Fr}_{0}(k)\right)$. Their sections on $U \in$ $S m / k$ will also be denoted by $\operatorname{Fr}_{m}\left(U, X \times T^{n}\right), \operatorname{Fr}\left(U, X \times T^{n}\right), \mathbb{Z}\left[\operatorname{Fr}_{m}\right]\left(U, X \times T^{n}\right)$, $\mathbb{Z} F_{m}\left(U, X \times T^{n}\right), \mathbb{Z}[\operatorname{Fr}]\left(U, X \times T^{n}\right), \mathbb{Z} F\left(U, X \times T^{n}\right)$ respectively. Notice that $X \times$ $\left(\mathbb{A}^{1}, \mathbb{G}_{m}\right)^{\wedge n}=\left(X \times \mathbb{A}^{n}, X \times\left(\mathbb{A}^{n}-0\right)\right)$.

There is an obvious functor spc:SmOp $\left(\operatorname{Fr}_{0}(k)\right) \rightarrow S h v_{\bullet}(S m / k)$ sending an object $(X, U) \in S m O p\left(\operatorname{Fr}_{0}(k)\right)$ to the pointed Nisnevich sheaf $X / U$. Observe that this functor preserves the monoidal product.

Recall from [GP1, Section 3] that for any integer $n \geqslant 0$, the endofunctor $\mathcal{F} r_{n}$ : $S h v_{\bullet}(S m / k) \rightarrow S h v_{\bullet}(S m / k)$ takes $\mathcal{F} \in S h v_{\bullet}(S m / k)$ to $\underline{\operatorname{Hom}}_{S h v_{\bullet}(S m / k)}\left(\mathbb{P}^{\wedge n}, \mathcal{F} \wedge\right.$ $\left.T^{n}\right)$. The stabilization in the canonical morphism $\sigma: \mathbb{P}^{\wedge 1} \rightarrow T$ leads to the endofunctor $\mathcal{F r}: S h v_{\bullet}(S m / k) \rightarrow S h v_{\bullet}(S m / k)$. It follows from [GP1, Section 3] that 
there are canonical isomorphisms between functors

$$
a_{n}: \operatorname{Fr}_{n} \cong \mathcal{F} r_{n} \circ s p c \text { and } a: \text { Fr } \cong \mathcal{F} r \circ s p c
$$

in the category of functors from $\operatorname{SmOp}\left(\operatorname{Fr}_{0}(k)\right)$ to $\left.S h v_{\bullet}(S m / k)\right)$.

$$
\text { 3. Presheaves } \operatorname{Fr}^{q f}(-, Y /(Y-S) \wedge T) \text { AND } \mathbb{Z F}^{q f}(-, Y /(Y-S) \wedge T)
$$

One of the main objectives of Sections 3,4 and 5 is to give an explicit description of the quotient presheaf $\mathbb{Z F}\left(-, X \times T^{n} \times \mathbb{A}^{1}\right) / \mathbb{Z F}\left(-, X \times T^{n} \times \mathbb{G}_{m}\right)$ on essentially smooth local henselian schemes. It turns out that this quotient presheaf coincides on such schemes with a subpresheaf $\mathbb{Z F}^{q f}\left(-, X \times T^{n+1}\right)$ of the presheaf $\mathbb{Z F}\left(-, X \times T^{n+1}\right)$ introduced in this section. More precisely, the canonical morphism $p:\left(\mathbb{A}^{1}, \emptyset\right) \rightarrow\left(\mathbb{A}^{1}, \mathbb{G}_{m}\right)$ in $\operatorname{SmOp}\left(\operatorname{Fr}_{0} k\right)$ induces a morphism of presheaves

$$
\bar{p}: \mathbb{Z F}\left(-, X \times T^{n} \times \mathbb{A}^{1}\right) / \mathbb{Z} \mathrm{F}\left(-, X \times T^{n} \times \mathbb{G}_{m}\right) \rightarrow \mathbb{Z} \mathrm{F}\left(-, X \times T^{n+1}\right) .
$$

Lemma 3.8(2), Lemma 5.2 and Corollary 5.3 imply that the morphism $\bar{p}$ is locally a monomorphism and its image coincides with the subpresheaf $\mathbb{Z F}^{q f}\left(-, X \times T^{n+1}\right)$ of the presheaf $\mathbb{Z F}\left(-, X \times T^{n+1}\right)$. Examples given in this section show that sections of this subpresheaf do not coincide with sections of $\mathbb{Z F}\left(-, X \times T^{n+1}\right)$ (even on the base field). Thus the morphism $\bar{p}$ is far from being a local isomorphism.

Notation 3.1. As recalled in Definition 2.5 the category $\operatorname{SmOp}\left(\mathrm{Fr}_{0}(k)\right)$ is symmetric monoidal. Let $(Y, Y-S) \in S m O p\left(\operatorname{Fr}_{0}(k)\right)$. Similarly to Notation 2.8 we shall write $\operatorname{Fr}_{m}(-, Y /(Y-S) \wedge T)$ (respectively $\operatorname{Fr}(-, Y /(Y-S) \wedge T), \mathbb{Z F}_{m}(-, Y /(Y-$ $S) \wedge T), \mathbb{Z F}(-, Y /(Y-S) \wedge T))$ to denote the value of the functor $\mathrm{Fr}_{m}$ (respectively Fr, $\left.\mathbb{Z} \mathrm{F}_{m}, \mathbb{Z F}\right)$ on the couple $(Y, Y-S) \wedge\left(\mathbb{A}^{1}, \mathbb{G}_{m}\right)=\left(Y \times \mathbb{A}^{1}, Y \times \mathbb{A}^{1}-S \times\{0\}\right)$.

Following Notation 3.1, a section of $\operatorname{Fr}_{m}(-, Y /(Y-S) \wedge T)$ on $U \in S m / k$ is given by a tuple

$$
\left(Z, W, \varphi_{1}, \ldots, \varphi_{m} ; g: W \rightarrow Y ; f: W \rightarrow \mathbb{A}^{1}\right),
$$

where $Z$ is a closed subset of $U \times \mathbb{A}^{m}$ finite over $U, W$ is an étale neighborhood of $Z$ in $U \times \mathbb{A}^{m}, \varphi_{1}, \ldots, \varphi_{m}, f$ are regular functions on $W, g$ is a regular map such that

$$
Z=Z\left(\varphi_{1}, \ldots, \varphi_{m}, f\right) \cap g^{-1}(S)
$$

The suspension map

$$
\Sigma: \operatorname{Fr}_{m}(-, Y /(Y-S) \wedge T) \rightarrow \operatorname{Fr}_{m+1}(-, Y /(Y-S) \wedge T)
$$

evaluated on $U \in S m / k$ sends

$$
\begin{aligned}
& \left(Z, W, \varphi_{1}, \ldots, \varphi_{m} ; g ; f\right) \text { to } \\
& \quad\left(Z \times\{0\}, W \times \mathbb{A}^{1}, \varphi_{1} \circ p r_{W}, \ldots, \varphi_{m} \circ p r_{W}, p r_{\mathbb{A}^{1}} ; g \circ p r_{W} ; f \circ p r_{W}\right) .
\end{aligned}
$$

For brevity, we write $\left(Z \times\{0\}, W \times \mathbb{A}^{1}, \varphi, t ; g ; f\right)$ for the latter framed correspondence.

Definition 3.2. Let $\operatorname{Fr}_{m}^{q f}(U, Y /(Y-S) \wedge T) \subset \operatorname{Fr}_{m}(U, Y /(Y-S) \wedge T)$ be the subset consisting of those elements $c$ for which there is an explicit framed correspondence $\left(Z, W, \varphi_{1}, \ldots, \varphi_{m} ; g ; f\right)$ representing $c$ such that the closed subset $Z\left(\varphi_{1}, \ldots, \varphi_{m}\right) \cap$ $g^{-1}(S) \subset W$ is quasi-finite over $U$. 
Example 3.3. Here are some simple examples of non quasi-finite framed correspondences:

- $\left(\{0\}, \mathbb{A}^{m}, t_{1}, t_{1}, \ldots, t_{m-1}, \mathbb{A}^{m} \rightarrow \operatorname{Spec}(k) ; t_{m}\right)$ belongs to $\operatorname{Fr}_{m}(\operatorname{Spec}(k), T) \backslash$ $\operatorname{Fr}_{m}^{q f}(\operatorname{Spec}(k), T)$;

- $\left(\{0\}, \mathbb{A}^{m}, t_{1}, t_{2}, t_{2}, . ., t_{m-1}, \mathbb{A}^{m} \rightarrow \operatorname{Spec}(k) ; t_{m}\right)$ belongs to $\operatorname{Fr}_{m}(\operatorname{Spec}(k), T) \backslash$ $\operatorname{Fr}_{m}^{q f}(\operatorname{Spec}(k), T)$

- $\left(\{0\}, \mathbb{A}^{m}, t_{1}, t_{2}, \ldots, t_{m-1}, t_{m-1}, \mathbb{A}^{m} \rightarrow \operatorname{Spec}(k) ; t_{m}\right)$ belongs to $\operatorname{Fr}_{m}(\operatorname{Spec}(k), T) \backslash$ $\operatorname{Fr}_{m}^{q f}(\operatorname{Spec}(k), T)$.

More generally, let $\varphi_{1}, \ldots, \varphi_{m-1} \in k\left[\mathbb{A}^{m}\right]=k\left[t_{1}, \ldots, t_{m}\right]$ be polynomials such that their common zero locus in $\mathbb{A}^{m}$ is an equidimensional closed subset of dimension 1. Let $f \in k\left[t_{1}, \ldots, t_{m}\right]$ be such that the common zero locus $Z$ of the functions $\varphi_{1}, \ldots, \varphi_{m-1}, f$ has dimension zero. Then

- $\left(Z, \mathbb{A}^{m}, \varphi_{1}, \ldots, \varphi_{m-1}, \mathbb{A}^{m} \rightarrow \operatorname{Spec}(k) ; f\right) \in \operatorname{Fr}_{m}(\operatorname{Spec}(k), T) \backslash \operatorname{Fr}_{m}^{q f}(\operatorname{Spec}(k), T)$.

Definition 3.4. Set $\operatorname{Fr}_{*}^{q f}(U, Y /(Y-S) \wedge T):=\bigsqcup_{m \geqslant 0} \operatorname{Fr}_{m}^{q f}(U, Y /(Y-S) \wedge T)$. Clearly, $\operatorname{Fr}_{*}^{q f}(-, Y /(Y-S) \wedge T)$ is a framed subpresheaf of the framed presheaf $\operatorname{Fr}_{*}(-, Y /(Y-S) \wedge T)$. Also, it is clear that the suspension $\Sigma$ takes $\operatorname{Fr}_{m}^{q f}(U, Y /(Y-$ $S) \wedge T)$ to $\operatorname{Fr}_{m+1}^{q f}(U, Y /(Y-S) \wedge T)$. Set,

$$
\begin{aligned}
\operatorname{Fr}^{q f}(-, Y /(Y-S) \wedge T):=\operatorname{colim}\left(\operatorname{Fr}_{0}^{q f}(-\right. & Y /(Y-S) \wedge T) \\
& \left.\stackrel{\Sigma}{\rightarrow} \operatorname{Fr}_{1}^{q f}(-, Y /(Y-S) \wedge T) \stackrel{\Sigma}{\rightarrow} \ldots\right) .
\end{aligned}
$$

By the very construction, $\operatorname{Fr}^{q f}(-, Y /(Y-S) \wedge T)$ is a pointed framed subpresheaf of the pointed framed presheaf $\operatorname{Fr}(-, Y /(Y-S) \wedge T)$.

Let $(Z, W, \varphi ; g ; f) \in \operatorname{Fr}_{m}(U, Y /(Y-S))$ be such that $Z=Z_{1} \sqcup Z_{2}$. Then $(Z, W, \varphi ; g ; f) \in \operatorname{Fr}_{m}^{q f}(U, Y /(Y-S))$ if and only if for $i, j=1,2$ and $j \neq i$ one has

$$
\left(Z_{i}, W \backslash Z_{j},\left.\varphi\right|_{W \backslash Z_{j}} ;\left.g\right|_{W \backslash Z_{j}} ;\left.f\right|_{W \backslash Z_{j}}\right) \in \operatorname{Fr}_{m}^{q f}(U, Y /(Y-S)) .
$$

This observation leads to the following

Definition 3.5. Let $Y \in S m / k$ and $S \subset Y$ be as in Definition 2.1. Let $U$ be a $k$-smooth scheme. Set,

$$
\mathbb{Z} \mathrm{F}_{m}^{q f}(U, Y /(Y-S) \wedge T):=\mathbb{Z}\left[\operatorname{Fr}_{m}^{q f}(U, Y /(Y-S) \wedge T)\right] / A,
$$

where $A$ is the subgroup generated by the elements

$$
\begin{aligned}
\left(Z \sqcup Z^{\prime}, W, \varphi ; g ; f\right)-\left(Z, W \backslash Z^{\prime},\left.\varphi\right|_{W \backslash Z^{\prime}} ;\left.g\right|_{W \backslash Z^{\prime}} ;\left.f\right|_{W \backslash Z^{\prime}}\right)- & \\
& -\left(Z^{\prime}, W \backslash Z,\left.\varphi\right|_{W \backslash Z} ;\left.g\right|_{W \backslash Z} ;\left.g\right|_{W \backslash Z}\right) .
\end{aligned}
$$

Set $\mathbb{Z F}_{*}^{q f}(U, Y /(Y-S) \wedge T)=\bigoplus_{m \geqslant 0} \mathbb{Z F}_{m}^{q f}(U, Y /(Y-S) \wedge T)$. The pairing (2.3) gives rise to a natural pairing $\mathbb{Z F}_{k}(V, U) \times \mathbb{Z F}_{m}^{q f}(U, Y /(Y-S)) \rightarrow \mathbb{Z F}_{k+m}^{q f}(U, Y /(Y-$ $S)$ ). The latter pairing makes $\mathbb{Z F}_{*}^{q f}(-, Y /(Y-S))$ a linear framed presheaf.

The external product (2.4) gives rise to an external product

$$
\left.\mathbb{Z F}_{m}^{q f}(U, Y /(Y-S)) \wedge T\right) \times \mathbb{Z F}_{n}(\mathrm{pt}, \mathrm{pt}) \stackrel{-{ }_{-}}{\longrightarrow} \mathbb{Z F}_{m+n}^{q f}(U, Y /(Y-S) \wedge T) .
$$


Definition 3.6. Set,

$$
\begin{aligned}
\mathbb{Z} \mathrm{F}^{q f}(-, Y /(Y-S) \wedge T)=\operatorname{colim}\left(\mathbb{Z F}_{0}^{q f}(-, Y /(Y-S) \wedge T)\right. & \\
& \left.\stackrel{\Sigma}{\rightarrow} \mathbb{Z F}_{1}^{q f}(-, Y /(Y-S) \wedge T) \stackrel{\Sigma}{\rightarrow} \ldots\right) .
\end{aligned}
$$

By the very construction, $\mathbb{Z F}^{q f}(-, Y /(Y-S) \wedge T)$ is a linear framed presheaf.

Definition 3.7. For $U \in S m / k$ let $\mathrm{F}_{m}(U, Y /(Y-S) \wedge T)$ be the subset of $\operatorname{Fr}_{m}(U, Y /(Y-S) \wedge T)$ consisting of the elements

$$
\left(Z, W, \varphi_{1}, \ldots, \varphi_{m} ; g ; f\right) \in \operatorname{Fr}_{m}(U, Y /(Y-S) \wedge T)
$$

such that $Z$ is connected. Clearly, this definition is consistent with Definition 2.4. Set,

$$
\mathrm{F}_{m}^{q f}(U, Y /(Y-S) \wedge T)=\mathrm{F}_{m}(U, Y /(Y-S) \wedge T) \cap \operatorname{Fr}_{m}^{q f}(U, Y /(Y-S) \wedge T) .
$$

Clearly, the set $\mathrm{F}_{m}^{q f}(U, Y /(Y-S) \wedge T) \backslash *$ is a free basis of the free abelian group $\mathbb{Z F}_{m}^{q f}(U, Y /(Y-S) \wedge T)$.

The following lemma immediately follows from definitions.

Lemma 3.8. (1) The set $\left(\mathrm{F}_{m}^{q f}(U, Y /(Y-S) \wedge T) \backslash *\right)$ is a free basis of $\mathbb{Z} \mathrm{F}_{m}^{q f}(U, Y /(Y-$ $S) \wedge T)$.

(2) The natural map $\mathbb{Z F}_{m}^{q f}(U, Y /(Y-S) \wedge T) \rightarrow \mathbb{Z F}_{m}(U, Y /(Y-S) \wedge T)$ is injective and identifies $\mathbb{Z F}_{m}^{q f}(U, Y /(Y-S) \wedge T)$ with a direct summand of $\mathbb{Z F}_{m}(U, Y /(Y$ $S) \wedge T)$. Therefore $\mathbb{Z F}^{q f}(-, Y /(Y-S) \wedge T)$ is a framed subpresheaf of the framed presheaf $\mathbb{Z} \mathrm{F}(-, Y /(Y-S) \wedge T)$.

Remark 3.9. However, the assignment $U \mapsto \mathrm{F}_{m}^{q f}(U, Y /(Y-S) \wedge T)$ is not a presheaf even on the category $S m / k$. Indeed, if $u \stackrel{i}{\rightarrow} U$ is a closed point and

$$
(Z, W, \varphi ; g) \in \mathrm{F}_{m}^{q f}(U, Y /(Y-S) \wedge T) \subseteq \operatorname{Fr}_{m}^{q f}(U, Y /(Y-S) \wedge T),
$$

then the support of $i^{*}(Z, W, \varphi ; g) \in \operatorname{Fr}_{m}^{q f}(u, Y /(Y-S) \wedge T)$ is the closed subset $Z_{u}$ in $\mathbb{A}_{u}^{m}$, but $Z_{u}$ is often not connected.

\section{Geometric lemmas}

In this section we prove a couple of geometric lemmas used in the following sections. All schemes in this section are supposed to be affine and noetherian. If $X$ is an affine noetherian scheme and $Z \subset X$ is a closed subset then by [G, 6.9] the henselization $X_{Z}^{h}$ of $X$ at $Z$ is an affine noetherian scheme. We start with a useful remark.

Remark 4.1. Let $W$ be a reduced local scheme with a closed point $w$. Let $S \subset W$ be a closed subset. Then $S$ with the reduced scheme structure is a local scheme which is connected and $w$ is the only closed point of $S$.

Furthermore, let $U$ be a reduced henselian local scheme with the closed point $u$. Let $S \subset W$ be a closed subset. Let $\pi: W \rightarrow U$ be a morphism such that $\pi(w)=u$ and let $\left.\pi\right|_{S}: S \rightarrow U$ be quasi-finite. By [Mi, Theorem I.4.2] $S$ is finite over $U$. Thus $S$ is reduced henselian local.

Let $S$ be a henselian local scheme and let $Z$ be a closed subset of $S$. Suppose $S_{Z}^{h}$ is the henselization of $S$ at $Z$. Then the canonical morphism $\operatorname{can}_{S, Z}: S_{Z}^{h} \rightarrow S$ is an isomorphism. Indeed, $\left(S, i d_{S}, i: Z \hookrightarrow S\right)$ is the initial object in the category 
of étale neighborhoods of $Z$ in $S$. Since $\operatorname{can}_{S, Z}: S_{Z}^{h} \rightarrow S$ is an isomorphism, the scheme $S_{Z}^{h}$ is noetherian henselian local.

Lemma 4.2. Let $V$ be an affine scheme, $Z \subset V$ be a closed connected subset, can $=$ $\operatorname{can}_{V, Z}: V_{Z}^{h} \rightarrow V$ be the henselization of $V$ at $Z$, and let $s: Z \rightarrow V_{Z}^{h}$ be the section of can over $Z$. Let $U$ be a regular local henselian scheme, $q: V \rightarrow U$ be a smooth morphism such that the morphism $\left.q\right|_{Z}: Z \rightarrow U$ is finite. Furthermore, suppose $Y \subset V_{Z}^{h}$ is a closed subset containing $s(Z)$, which is quasi-finite over $U$. Then $\left.\operatorname{can}\right|_{Y}: Y \rightarrow V$ is a closed embedding, can $(Y)$ contains $Z$ and $\operatorname{can}^{-1}(\operatorname{can}(Y))=Y$.

Proof. Since $Z$ is finite over the local henselian $U$ and $Z$ is connected, it is local and henselian. Since $Z$ is local, then so is the scheme $W=V_{Z}^{h}$. By Remark 4.1 the scheme $Y$ with the reduced scheme structure is local, connected and finite over $U$. Hence the set $Y_{1}=\operatorname{can}(Y)$ is closed in $V$ and finite over $U$ and contains $Z$. So $Y_{1}$ with the reduced scheme structure is a local henselian scheme.

We claim that there is a canonical isomorphism of schemes between the henselization $\left(Y_{1}\right)_{Z}^{h}$ of $Y_{1}$ at $Z$ and the scheme $\operatorname{can}^{-1}\left(Y_{1}\right)$. This claim is a partial case of [FP, Lemma 5.8]. In order to apply that lemma, our scheme $V$ is replaced by $S$, our scheme $Y_{1}$ is replaced by $S^{\prime}$, and our local scheme $Z$ is replaced by $T$. Within this notation the scheme $T \times_{S} S^{\prime}$ from that lemma is just our scheme $Z$. Also, in this case the canonical scheme isomorphism $\left(T \times_{S} S^{\prime}\right)^{h} \rightarrow T^{h} \times_{S} S^{\prime}$ from that lemma is the isomorphism of schemes $\left(Y_{1}\right)_{Z}^{h} \rightarrow \mathrm{can}^{-1}\left(Y_{1}\right)$. Hence the pair $\left(\operatorname{can}^{-1}\left(Y_{1}\right), s(Z)\right)$ is the henselization $\left(Y_{1}\right)_{Z}^{h}$ of $Y_{1}$ at $Z$. Thus, by Remark 4.1 the morphism $p_{1}=\operatorname{can}_{\operatorname{can}^{-1}\left(Y_{1}\right)}: \operatorname{can}^{-1}\left(Y_{1}\right) \rightarrow Y_{1}$ is an isomorphism of schemes. Since the scheme $Y_{1}$ is reduced, so is the scheme $\operatorname{can}^{-1}\left(Y_{1}\right)$.

Clearly, $Y$ regarded with the reduced scheme structure is a closed subscheme of the scheme $\operatorname{can}^{-1}\left(Y_{1}\right)$. Let $i: Y \rightarrow \operatorname{can}^{-1}\left(Y_{1}\right)$ be the inclusion. Then the ring map $\left(p_{1} \circ i\right)^{*}: \Gamma\left(Y_{1}, \mathcal{O}_{Y_{1}}\right) \rightarrow \Gamma\left(Y, \mathcal{O}_{Y}\right)$ is surjective. On the other hand, this ring morphism is injective, because both schemes are reduced affine and the morphism $p_{1} \circ i$ is surjective. Hence $p_{1} \circ i: Y \rightarrow Y_{1}$ is an isomorphism of schemes. Thus can $\left.\right|_{Y}: Y \rightarrow V$ is a closed embedding.

Lemma 4.3. Under the assumptions of Lemma 4.2 supoose $Y \subset V$ is a closed connected subset containing $Z$ and is finite over $U$. Then there is a unique section $t: Y \rightarrow V_{Z}^{h}$ of the morphism can $: V_{Z}^{h} \rightarrow V$ and $t(Y)=\operatorname{can}^{-1}(Y)$ contains $s(Z)$.

Proof. Consider the closed subset $Y \subset V$ with the reduced scheme structure. Since $U$ is local henselian, then so is the scheme $Y$. Using [FP, Lemma 5.8] similarly to the proof of Lemma 4.2, we conclude that the scheme $\operatorname{can}^{-1}(Y)$ is the henselization of $Y$ at $Z$. By Remark 4.1 the morphism $p=\left.\operatorname{can}\right|_{\operatorname{can}^{-1}(Y)}: \operatorname{can}^{-1}(Y) \rightarrow Y$ is an isomorphism of schemes. Set $t=i n \circ p^{-1}: Y \rightarrow V_{Z}^{h}$, where in $: \operatorname{can}^{-1}(Y) \hookrightarrow V_{Z}^{h}$ is the closed embedding. Clearly, $t$ is a section of can over $Y$. If $t^{\prime}: Y \rightarrow V_{Z}^{h}$ is another section of can, then $t^{\prime}=t$.

\section{The Linear Framed PREsheaf $\mathbb{Z F}^{q f}\left(X \times T^{n+1}\right)$}

Let $X$ be a $k$-smooth variety and let $Y=X \times \mathbb{A}^{n}, S=X \times\{0\}$. Following Notations 2.8, 3.1 and Definitions 3.2, 3.5, 3.6, we shall write $\operatorname{Fr}^{q f}\left(-, X \times T^{n+1}\right)$ and $\mathbb{Z F}^{q f}\left(-, X \times T^{n+1}\right)$ to denote the presheaves $\operatorname{Fr}^{q f}(-,(Y /(Y-S)) \wedge T)$ and $\mathbb{Z F}^{q f}(-,(Y /(Y-S)) \wedge T)$ respectively. We also have the following equalities:

$$
\mathbb{Z F}\left(-, X \times T^{n+1}\right)=\mathbb{Z F}(-,(Y /(Y-S)) \wedge T)
$$


and

$$
\operatorname{Fr}\left(-, X \times T^{n+1}\right)=\operatorname{Fr}(-,(Y /(Y-S)) \wedge T) .
$$

We also have equalities of framed presheaves as follows:

$$
\begin{aligned}
& \diamond \operatorname{Fr}_{*}^{q f}\left(-, X \times T^{n+1}\right)=\operatorname{Fr}_{*}^{q f}(-,(Y /(Y-S)) \wedge T), \\
& \diamond \operatorname{Fr}_{*}\left(-, X \times T^{n+1}\right)=\operatorname{Fr}_{*}(-,(Y /(Y-S)) \wedge T), \\
& \diamond \mathbb{Z F}_{*}^{q f}\left(-, X \times T^{n+1}\right)=\mathbb{Z F}_{*}^{q f}(-,(Y /(Y-S)) \wedge T), \\
& \diamond \mathbb{Z F}_{*}\left(-, X \times T^{n+1}\right)=\mathbb{Z}_{*}(-,(Y /(Y-S)) \wedge T),
\end{aligned}
$$

Finally, we have equalities of pointed sets:

$$
\begin{aligned}
& \diamond \mathrm{F}_{m}\left(U, X \times T^{n+1}\right)=\mathrm{F}_{m}(U,(Y /(Y-S)) \wedge T), \\
& \diamond \mathrm{F}_{m}^{q f}\left(U, X \times T^{n+1}\right)=\mathrm{F}_{m}^{q f}(U,(Y /(Y-S)) \wedge T) .
\end{aligned}
$$

Specifying definitions of Section 3, a section of $\operatorname{Fr}_{m}\left(-,\left(X \times T^{n+1}\right)\right)$ on $U \in S m / k$ is a tuple

$$
c=\left(Z, W, \varphi_{1}, \ldots, \varphi_{m} ; h: W \rightarrow X \times \mathbb{A}^{n} ; f: W \rightarrow \mathbb{A}^{1}\right),
$$

where $Z$ is a closed subset of $U \times \mathbb{A}^{m}$ finite over $U, W$ is an étale neighborhood of $Z$ in $\mathbb{A}^{m} \times U, \varphi_{1}, \ldots, \varphi_{m}, f$ are regular functions on $W, h=\left(g, \varphi_{m+1}, \ldots, \varphi_{m+n}\right)$ : $W \rightarrow X \times \mathbb{A}^{n}$ is a regular map such that

$$
Z=Z\left(\varphi_{1}, \ldots, \varphi_{m}, f\right) \cap h^{-1}(X \times\{0\})=Z\left(\varphi_{1}, \ldots, \varphi_{m}, f, \varphi_{m+1}, \ldots, \varphi_{m+n}\right) .
$$

The section $c$ belongs to $\operatorname{Fr}_{m}^{q f}\left(U, X \times T^{n+1}\right)$ if and only if the vanishing locus $Z\left(\varphi_{1}, \ldots, \varphi_{m}, \varphi_{m+1}, \ldots, \varphi_{m+n}\right)$ is quasi-finite over $U$.

The section $c$ belongs to $\mathrm{F}_{m}\left(U, X \times T^{n+1}\right)$ if and only if the set $Z$ is connected. The section $c$ is in $\mathrm{F}_{m}^{q f}\left(U, X \times T^{n+1}\right)$ if and only if the set $Z$ is connected and the vanishing locus $Z\left(\varphi_{1}, \ldots, \varphi_{m}, \varphi_{m+1}, \ldots, \varphi_{m+n}\right)$ is quasi-finite over $U$.

Recall that the suspension morphism $\Sigma: \operatorname{Fr}_{m}\left(-, X \times T^{n+1}\right) \rightarrow \operatorname{Fr}_{m+1}(-, X \times$ $\left.T^{n+1}\right)$ sends a tuple $\left(Z, W, \varphi_{1}, \ldots, \varphi_{m} ; g ; \varphi_{m+1}, \ldots, \varphi_{m+n} ; f\right)$ to the tuple $(Z \times$ $\left.\{0\}, W \times \mathbb{A}^{1}, \varphi_{1}, \ldots, \varphi_{m}, t ; g ; \varphi_{m+1}, \ldots, \varphi_{m+n} ; f\right)$.

Notation 5.1. In what follows, we write $\left(Z, W, \varphi_{1}, \ldots, \varphi_{m}, \varphi_{m+1}, \ldots, \varphi_{m+n} ; f\right.$ : $\left.W \rightarrow \mathbb{A}^{1}, g: W \rightarrow X\right)$ to denote $\left(Z, W, \varphi_{1}, \ldots, \varphi_{m} ; g: W \rightarrow X ; \varphi_{m+1}, \ldots, \varphi_{m+n} ; f:\right.$ $W \rightarrow \mathbb{A}^{1}$ ) for the convenience of computations.

Let $m \geqslant 0$ be an integer. Using Construction 2.6 the canonical morphism $\left(\mathbb{A}^{1}, \emptyset\right) \rightarrow\left(\mathbb{A}^{1}, \mathbb{G}_{m}\right)$ in $\operatorname{SmOp}\left(\operatorname{Fr}_{0}(k)\right.$ induces a morphism in $\left.S h v_{\bullet}(S m / k)\right)$ :

$$
p: \operatorname{Fr}_{m}\left(-, X \times T^{n} \times \mathbb{A}^{1}\right) \rightarrow \operatorname{Fr}_{m}\left(-, X \times T^{n+1}\right) .
$$

This morphism sends a tuple $\left(Z, W, \varphi_{1}, \ldots, \varphi_{m}, \varphi_{m+1}, \ldots, \varphi_{m+n} ; f ; g\right)$ to the tuple $\left(Z^{\prime}, W, \varphi_{1}, \ldots, \varphi_{m}, \varphi_{m+1}, \ldots, \varphi_{m+n}, \varphi_{m+n+1} ; g\right)$ with $Z^{\prime}=Z \cap Z(f), \varphi_{m+n+1}=f$.

Lemma 5.2. If $U$ is essentially $k$-smooth local henselian, then the image of the map $p: \operatorname{Fr}_{m}\left(U, X \times T^{n} \times \mathbb{A}^{1}\right) \rightarrow \operatorname{Fr}_{m}\left(U, X \times T^{n+1}\right)$ is contained in $\operatorname{Fr}_{m}^{q f}(U, X \times$ $\left.T^{n+1}\right)$. Moreover, for any $m \geqslant 0$ the map pends $\mathrm{F}_{m}\left(U, X \times T^{n} \times \mathbb{A}^{1}\right)$ to the set $\mathrm{F}_{m}^{q f}\left(U, X \times T^{n+1}\right)$. Finally, the square of pointed sets

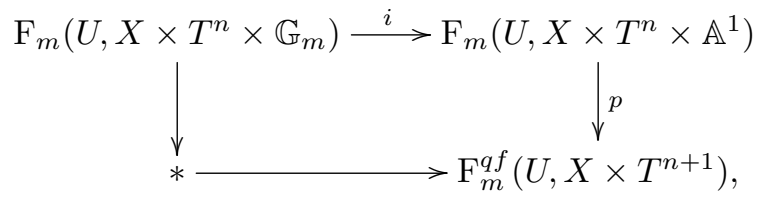

is a pushout square. Here $* \in \mathrm{F}_{m}\left(U, X \times T^{n+1}\right)$ is the empty framed correspondence. 
Proof. The first assertion is obvious. To prove the second one, take an element

$$
c=\left(Y, W, \varphi_{1}, \ldots, \varphi_{m+n}, f: W \rightarrow \mathbb{A}^{1} ; g: W \rightarrow X\right) \in \mathrm{F}_{m}\left(U, X \times T^{n} \times \mathbb{A}^{1}\right)
$$

with $W=\left(\mathbb{A}^{m} \times U\right)_{Y}^{h}$. Then $p(c)=\left(Z, W, \varphi_{1}, \ldots, \varphi_{m+n+1} ; g\right)$, where $Z=Y \cap Z(f)$, $\varphi_{m+n+1}=f$.

Since $Y$ is connected and finite over the henselian $U$, then $Y$ is henselian and local. Hence $W$ is local. By Remark 4.1 the closed subset $Z$ is connected, whence the second assertion of the lemma.

To prove the third assertion, it sufficient to construct a section

$$
s: \mathrm{F}_{m}^{q f}\left(U, X \times T^{n+1}\right) \backslash * \rightarrow \mathrm{F}_{m}\left(U, X \times T^{n} \times \mathbb{A}^{1}\right) \backslash \mathrm{F}_{m}\left(U, X \times T^{n} \times \mathbb{G}_{m}\right)
$$

of $p$ and check that the map $p$ is injective on the complement of $\mathrm{F}_{m}\left(U, X \times T^{n} \times \mathbb{G}_{m}\right)$.

We construct $s$ as follows. Take $c=\left(Z, \mathbb{A}^{m} \times U \stackrel{c a n}{\longleftarrow} W, \varphi_{1}, \ldots, \varphi_{m+n+1} ; g\right)$ from $\mathrm{F}_{m}^{q f}\left(U, X \times T^{n+1}\right)$ with $Z$ non-empty and with $W=\left(\mathbb{A}^{m} \times U\right)_{Z}^{h}$. Since $Z$ is connected and finite over the local henselian $U$, the scheme $W$ is local. Set

$$
s(c)=\left(\operatorname{can}(Y), \mathbb{A}^{m} \times U \stackrel{c a n}{\longleftarrow} W, \varphi_{1}, \ldots, \varphi_{m+n} ; \varphi_{m+n+1}: W \rightarrow \mathbb{A}^{1} ; g: W \rightarrow X\right),
$$

where $Y=Z\left(\varphi_{1}, \ldots, \varphi_{m+n}\right) \subset W$. The set $Y$ is quasi-finite over $U$, because $c \in \mathrm{F}_{m}^{q f}\left(U, X \times T^{n+1}\right)$. By Remark 4.1 the set $Y$ is finite over $U$ and connected and local. By Lemma 4.2 the morphism $\left.\operatorname{can}\right|_{Y}: Y \rightarrow \mathbb{A}^{m} \times U$ is a closed embedding and $\operatorname{can}^{-1}(\operatorname{can}(Y))=Y$. Thus $s(c) \in \mathrm{F}_{m}\left(U, X \times T^{n+1} \times \mathbb{A}^{1}\right)$. Clearly, $p(s(c))=c$.

Now check the required injectivity for $p$. Let $c^{\prime}=\left(Y^{\prime}, W^{\prime}, \varphi_{1}^{\prime}, \ldots, \varphi_{m+n}^{\prime}, f^{\prime}\right.$ : $\left.W^{\prime} \rightarrow \mathbb{A}^{1} ; g^{\prime}: W^{\prime} \rightarrow X\right)$ and $c^{\prime \prime}=\left(Y^{\prime \prime}, W^{\prime \prime}, \varphi_{1}^{\prime \prime}, \ldots, \varphi_{m+n}^{\prime \prime}, f^{\prime \prime}: W^{\prime \prime} \rightarrow \mathbb{A}^{1} ; g^{\prime \prime}:\right.$ $\left.W^{\prime \prime} \rightarrow X\right)$ in $\mathrm{F}_{m}\left(U, X \times T^{n} \times \mathbb{A}^{1}\right)$ be two elements with $W^{\prime}=\left(\mathbb{A}^{m} \times U\right)_{Y^{\prime}}^{h}$ and $W^{\prime \prime}=\left(\mathbb{A}^{m} \times U\right)_{Y^{\prime \prime}}^{h}$. Let $c a n^{\prime}: W^{\prime} \rightarrow \mathbb{A}^{m} \times U$ be the canonical morphism and let $s^{\prime}: Y^{\prime} \rightarrow W^{\prime}$ be the section of $c a n^{\prime}$.

Suppose that $p(c)=p\left(c^{\prime}\right)$ and the support $Z=Y^{\prime} \cap Z\left(f^{\prime}\right)=Y^{\prime \prime} \cap Z\left(f^{\prime \prime}\right)$ is non-empty. We must check that $c^{\prime}=c^{\prime \prime}$. The element $p\left(c^{\prime}\right)$ is of the form

$$
\left(Z, \mathbb{A}^{m} \times U \stackrel{\text { can }}{\longleftarrow} W, \varphi_{1}, \ldots, \varphi_{m+n+1} ; g\right),
$$

where $Z=Y^{\prime} \cap Z\left(f^{\prime}\right), W=\left(\mathbb{A}^{m} \times U\right)_{Z}^{h}, \varphi_{i}=\left.\varphi_{i}\right|_{W}, g=\left.g\right|_{W}$. Consider the canonical morphism $\operatorname{can}_{1}: W \rightarrow W^{\prime}$. It is the henselization of $W^{\prime}$ at $s^{\prime}\left(Y^{\prime}\right)$. Note that $s^{\prime}\left(Y^{\prime}\right) \subset W^{\prime}$ is a closed subset containing $s^{\prime}(Z)$. Moreover, $Y^{\prime}$ is finite over the henselian $U$ and connected. By Lemma 4.3 there is a unique section $t^{\prime}: Y^{\prime} \rightarrow W$ of the morphism $c a n_{1}$, and $t^{\prime}\left(Y^{\prime}\right)=\operatorname{can}_{1}^{-1}\left(Y^{\prime}\right)$ contains $s(Z)$, where $s: Z \rightarrow W$ is the section of $\operatorname{can}_{1}$ (the morphism $s$ is also the section of $c a n=\operatorname{can}^{\prime} \circ \operatorname{can}_{1}$ ). These arguments imply an equality

$$
\begin{aligned}
c^{\prime}=\left(Y^{\prime}, W,\left.\varphi_{1}^{\prime}\right|_{W}, \ldots,\left.\varphi_{m+n}^{\prime}\right|_{W},\left.f^{\prime}\right|_{W}: W \rightarrow \mathbb{A}^{1} ;\left.g^{\prime}\right|_{W}: W \rightarrow X\right) & \\
& \in \mathrm{F}_{m}\left(U, X \times T^{n} \times \mathbb{A}^{1}\right) .
\end{aligned}
$$

For the same reason one has an equality

$$
\begin{aligned}
c^{\prime \prime}=\left(Y^{\prime \prime}, W,\left.\varphi_{1}^{\prime \prime}\right|_{W}, \ldots,\left.\varphi_{m+n}^{\prime \prime}\right|_{W},\left.f^{\prime \prime}\right|_{W}: W \rightarrow \mathbb{A}^{1} ;\left.g^{\prime \prime}\right|_{W}: W \rightarrow X\right) & \\
& \in \mathrm{F}_{m}\left(U, X \times T^{n} \times \mathbb{A}^{1}\right) .
\end{aligned}
$$

Since $W$ is the henselization of $\mathbb{A}^{m} \times U$ at $Z$ and $p\left(c^{\prime}\right)=p\left(c^{\prime \prime}\right)$, one has equalities $\left.\varphi_{i}^{\prime}\right|_{W}=\left.\varphi_{i}^{\prime \prime}\right|_{W}$ for $i=1, \ldots, m+n,\left.f^{\prime}\right|_{W}=f_{W}^{\prime \prime}$ and $\left.g^{\prime}\right|_{W}=\left.g^{\prime \prime}\right|_{W}$. Hence $Y^{\prime}=Y^{\prime \prime}$ and, moreover, $c^{\prime}=c^{\prime \prime}$ in $\mathrm{F}_{m}\left(U, X \times T^{n} \times \mathbb{A}^{1}\right)$. The desired injectivity is proved. The section $s$ is constructed above and our lemma follows. 
Corollary 5.3. For any integer $n \geqslant 0$ the natural morphism

$$
\alpha_{*}: \mathbb{Z} \mathrm{F}_{*}\left(X \times T^{n} \times \mathbb{A}^{1}\right) / \mathbb{Z F}_{*}\left(X \times T^{n} \times \mathbb{G}_{m}\right) \rightarrow \mathbb{Z F}_{*}^{q f}\left(X \times T^{n+1}\right)
$$

of $\mathbb{Z} \mathrm{F}_{*}(k)$-presheaves is an isomorphism locally for the Nisnevich topology. As a consequence, the natural morphism

$$
\alpha: \mathbb{Z} \mathrm{F}\left(X \times T^{n} \times \mathbb{A}^{1}\right) / \mathbb{Z} \mathrm{F}\left(X \times T^{n} \times \mathbb{G}_{m}\right) \rightarrow \mathbb{Z F}^{q f}\left(X \times T^{n+1}\right)
$$

of $\mathbb{Z} \mathrm{F}_{*}(k)$-presheaves is an isomorphism locally for the Nisnevich topology.

We are now in a position to prove Theorem 1.4.

Proof of Theorem 1.4. The theorem is implied by Definitions 3.6, 3.7 and Corollary 5.3 .

We refer the reader to [GP2] for the definition of quasi-stability of framed presheaves.

Lemma 5.4. Let $k$ be an infinite perfect field. Let $A$ and $B$ be linear framed presheaves such that the cohomology presheaves of the complexes $C_{*}(A)$ and $C_{*}(B)$ are quasi-stable. Let $\alpha: A \rightarrow B$ be a morphism of linear framed presheaves, which is an isomorphism locally in the Nisnevich topology. Then the morphism

$$
C_{*}(\alpha): C_{*}(A) \rightarrow C_{*}(B)
$$

is a quasi-isomorphism locally in the Nisnevich topology.

Proof. By assumption the map of the Eilenberg-Mac Lane spectra $\alpha: E M(A) \rightarrow$ $\operatorname{EM}(B)$ is a local weak equivalence. Hence the induced map $\alpha: E M\left(C_{*}(A)\right) \rightarrow$ $E M\left(C_{*}(B)\right)$ is a motivic weak equivalence of $S^{1}$-spectra. By assumption, the presheaves of stable homotopy groups of the spectra $\operatorname{EM}\left(C_{*}(A)\right), E M\left(C_{*}(B)\right)$ are radditive, quasi-stable and $\mathbb{A}^{1}$-invariant (see [Voe10] for the definition of radditivity).

Let $E M\left(C_{*}(A)\right)_{f}, E M\left(C_{*}(B)\right)_{f}$ be fibrant replacements of $E M\left(C_{*}(A)\right)$ and $E M\left(C_{*}(B)\right)$ in the level injective model structure of $S^{1}$-spectra. Then they are motivically fibrant spectra by $[\mathrm{GP} 1,7.4]$. Hence the stable motivic equivalence of $S^{1}$-spectra $\alpha_{f}: \operatorname{EM}\left(C_{*}(A)\right)_{f} \rightarrow E M\left(C_{*}(B)\right)_{f}$ is a Nisnevich local weak equivalence of $S^{1}$-spectra. Thus the morphism of complexes $C_{*}(\alpha): C_{*}(A) \rightarrow C_{*}(B)$ is a quasi-isomorphism locally in the Nisnevich topology.

We are now in a position to prove a statement which is necessary for the proof of Theorem 1.3.

Proposition 5.5. Let $k$ be an infinite perfect field. Then the morphism

$$
C_{*}(\alpha): C_{*} \mathbb{Z F}\left(X \times T^{n} \times \mathbb{A}^{1}\right) / C_{*} \mathbb{Z F}\left(X \times T^{n} \times \mathbb{G}_{m}\right) \rightarrow C_{*} \mathbb{Z F}^{q f}\left(X \times T^{n+1}\right)
$$

is a quasi-isomorphism locally in the Nisnevich topology.

Proof. The morphism $\alpha: \mathbb{Z} \mathrm{F}\left(X \times T^{n} \times \mathbb{A}^{1}\right) / \mathbb{Z F}\left(X \times T^{n} \times \mathbb{G}_{m}\right) \rightarrow \mathbb{Z F}^{q f}\left(X \times T^{n+1}\right)$ is a morphism of linear framed presheaves. Set $A=\mathbb{Z F}\left(X \times T^{n} \times \mathbb{A}^{1}\right) / \mathbb{Z F}(X \times$ $\left.T^{n} \times \mathbb{G}_{m}\right)$ and $B=\mathbb{Z F}^{q f}\left(X \times T^{n+1}\right)$. Then the cohomology presheaves of the complexes $C_{*}(A)$ and $C_{*}(B)$ are quasi-stable by the construction of $A$ and $B$. Now Theorem 1.4 and Lemma 5.4 imply the claim. 
Thus we have computed the complex $C_{*} \mathbb{Z F}\left(X \times T^{n} \times \mathbb{A}^{1}\right) / C_{*} \mathbb{Z} \mathrm{F}\left(X \times T^{n} \times \mathbb{G}_{m}\right)$ locally in the Nisnevich topology as the complex $C_{*} \mathbb{Z F}^{q f}\left(X \times T^{n+1}\right)$. Our next goal is to show that the latter complex is quasi-isomorphic to $C_{*} \mathbb{Z F}\left(X \times T^{n+1}\right)$ locally in the Zariski topology verifying Theorem 1.5. The next two sections are dedicated to this theorem.

We finish this section with the following remark justifying the use of property of quasi-stability of linear framed presheaves.

Remark 5.6. Lemma 5.4 is also true if the condition "the cohomology presheaves of the complexes $C_{*}(A)$ and $C_{*}(B)$ are quasi-stable" is replaced by the condition "the cohomology presheaves of the complexes $C_{*}(A)$ and $C_{*}(B)$ are stable": the morphism $C_{*}(\alpha): C_{*}(A) \rightarrow C_{*}(B)$ is a quasi-isomorphism locally in the Nisnevich topology.

However, we can not apply this for the proof of Proposition 5.5. Indeed, the cohomology presheaves of the complex

$$
C_{*} \mathbb{Z F}\left(X \times T^{n} \times \mathbb{A}^{1}\right) / C_{*} \mathbb{Z} \mathrm{F}\left(X \times T^{n} \times \mathbb{G}_{m}\right)
$$

are quasi-stable only. And it is not readily apparent that they are stable.

\section{A filtration on $\mathbb{Z F}_{n}\left(-, X \times T^{n+1}\right)$}

We start with the following

Definition 6.1. Let $U \in S m / k$ be an affine variety and let $c=(Z, W, \varphi ; g) \in$ $\operatorname{Fr}_{m}\left(U, X \times T^{n+1}\right)$ be a framed correspondence. A finite collection of polynomials $F_{1}, \ldots, F_{r} \in k\left[\mathbb{A}^{m+n+1} \times U\right]$ is said to be $c$-defining if for every point $u \in U$ there is $i \in\{1,2 \ldots, r\}$ such that

$\diamond$ the polynomial $F_{i}(-, u) \in k(u)\left[\mathbb{A}^{m+n+1}\right]$ is nonzero,

$\diamond \varphi\left(W_{u}\right) \subseteq Z\left(F_{i}(-, u)\right)$ in $\mathbb{A}_{u}^{m+n+1}$.

Note that if a finite set of polynomials $F_{1}, \ldots, F_{r} \in k\left[\mathbb{A}^{m+n+1} \times U\right]$ is $c$-defining, where $c=(Z, W, \varphi ; g)=\left(Z_{1} \sqcup Z_{2}, W, \varphi ; g\right)$, then the same collection of polynomials is $\left(Z_{1}, W-Z_{2}, \varphi ; g\right)$-defining and is $\left(Z_{2}, W-Z_{1}, \varphi ; g\right)$-defining respectively.

Remark 6.2. We want to explain how we use a finite $c$-defining set of polynomials $F_{1}, \ldots, F_{r} \in k\left[\mathbb{A}^{m+n+1} \times U\right]$. Take an integer $d$ strictly greater than the degrees of all $F_{i}$-s. Lemma 7.3 below gives rise to a "homotopy"

$$
h_{s}^{d}(c) \in \operatorname{Fr}_{n}\left(U \times \mathbb{A}^{1}, X \times T^{n+1}\right)
$$

between $h_{0}^{d}(c)=c \in \operatorname{Fr}_{n}\left(U, X \times T^{n+1}\right)$ and $h_{1}^{d}(c)=t_{d}(c) \in \operatorname{Fr}_{n}^{q f}\left(U, X \times T^{n+1}\right)$. Moreover, if $c \in \operatorname{Fr}_{m}^{q f}\left(U, X \times T^{n+1}\right)$, then $h_{s}^{d}(c) \in \operatorname{Fr}_{m}^{q f}\left(U \times \mathbb{A}^{1}, X \times T^{n+1}\right)$. Finally, if $Z$ is the support of $c$, then $Z \times \mathbb{A}^{1}$ is the support of $h_{s}^{d}(c)$.

The following lemma is crucial in our analysis.

Lemma 6.3. Let $m, n \geqslant 0$ and $Y$ an affine (possibly non-irreducible) $k$-variety. Let $W \rightarrow \mathbb{A}^{m} \times Y$ be an étale morphism and $\psi: W \rightarrow \mathbb{A}^{m+n+1} \times Y$ be a morphism of $Y$-schemes. Then there is a finite set of polynomials $F_{1}, \ldots, F_{r} \in k\left[\mathbb{A}^{m+n+1} \times Y\right]$ such that for every point $y \in Y$ there is $i \in\{1,2 \ldots, r\}$ such that

$\diamond$ the polynomial $F_{i}(-, y) \in k(y)\left[\mathbb{A}^{m+n+1}\right]$ is nonzero and

$\diamond \psi\left(W_{y}\right) \subseteq Z\left(F_{i}(-, u)\right)$ in $\mathbb{A}_{u}^{m+n+1}$. 
Proof. We proceed by induction in the dimension of $Y$. If $\operatorname{dim}(Y)=0$ then there is nothing to prove. Now suppose $\operatorname{dim}(Y)>0$. Let $Y_{1}, \ldots, Y_{l}$ be all irreducible components of $Y$. For an index $i$ from $\{1, \ldots, l\}$ take the restriction of the map $\left.\psi\right|_{W_{Y_{i}}}: W_{Y_{i}} \rightarrow \mathbb{A}^{m+n+1} \times Y_{i}$ to $Y_{i}$. The dimension of $W_{Y_{i}}$ is $m+\operatorname{dim}\left(Y_{i}\right)$. Thus the closure of its image is contained in the zero locus $Z\left(\bar{F}_{(i)}\right)$ of a non-zero polynomial $\bar{F}_{(i)} \in k\left[\mathbb{A}^{m+n+1} \times Y_{i}\right]$. Since $Y_{i}$ is closed in the affine variety $Y$, the polynomial $\bar{F}_{i}$ can be extended to a polynomial $F_{i} \in k\left[\mathbb{A}^{m+n+1} \times Y\right]$. Let $V \subset Y$ be an open subset consisting of those $y \in Y$ such that there is $i$ with $0 \neq F_{i}(-, y)$ in $k(y)\left[\mathbb{A}^{m+n+1}\right]$. Let $Y^{\prime}=Y-V$.

By construction, $V$ has a non-empty intersection with every irreducible component of $Y$. Thus $\operatorname{dim}\left(Y^{\prime}\right)<\operatorname{dim}(Y)$. By the inductive assumption there are polynomials $\bar{F}_{l+1}, \ldots, \bar{F}_{r}$ in $k\left[\mathbb{A}^{m+n+1} \times Y^{\prime}\right]$ such that for every point $y \in Y^{\prime}$ there is $j \in\{l+1, \ldots, r\}$ with each polynomial $\bar{F}_{j}(-, y) \in k(y)\left[\mathbb{A}^{m+n+1}\right]$ nonzero and $\psi\left(W_{y}\right) \subseteq Z\left(\bar{F}_{j}(-, y)\right)$ in $\mathbb{A}_{y}^{m+n+1}$. Since $Y^{\prime}$ is closed in the affine $Y$ for any $j$, the polynomial $\bar{F}_{j}$ can be extended to a polynomial $F_{j}$ in $k\left[\mathbb{A}^{m+n+1} \times Y\right]$. Clearly, the set of polynomials $F_{i}$, where $i \in\{1,2, \ldots, r\}$, are the desired polynomials for $Y$.

The preceding lemma has the following

Corollary 6.4. Let $U \in S m / k$ be an affine variety and let $c=(Z, W, \varphi ; g) \in$ $\operatorname{Fr}_{m}\left(U, X \times T^{n+1}\right)$ be a framed correspondence. Then there exists a c-defining set of polynomials $F_{1}, \ldots, F_{r} \in k\left[\mathbb{A}^{m+n+1} \times U\right]$.

Moreover, suppose $f: V \rightarrow U$ is a morphism of $k$-smooth affine varieties and $F_{1}, \ldots, F_{r} \in k\left[\mathbb{A}^{m+n+1} \times U\right]$ is a c-defining set, then $f^{*}\left(F_{1}\right), \ldots, f^{*}\left(F_{r}\right) \in$ $k\left[\mathbb{A}^{m+n+1} \times V\right]$ is a $f^{*}(c)$-defining set.

Let $U \in S m / k$ be an affine variety. Let $d>0$. Define $\operatorname{Fr}_{m}^{<d}\left(U, X \times T^{n+1}\right)$ as a subset of $\operatorname{Fr}_{m}\left(U, X \times T^{n+1}\right)$ consisting of those $c=(Z, W, \varphi ; g) \in \operatorname{Fr}_{m}\left(U, X \times T^{n+1}\right)$ for which there exists a $c$-defining set $F_{1}, \ldots F_{r} \in k\left[\mathbb{A}^{m+n+1} \times U\right]$ with $\operatorname{deg} F_{i}<d$ for all $i=1, \ldots, r$. Set,

$$
\left(\operatorname{Fr}_{m}^{q f}\right)^{<d}\left(U, X \times T^{n+1}\right):=\operatorname{Fr}_{m}^{q f}\left(U, X \times T^{n+1}\right) \cap \operatorname{Fr}_{m}^{<d}\left(U, X \times T^{n+1}\right) .
$$

Corollary 6.4 shows that each of these filtrations is stable under pullbacks. Hence one has the following obvious

Lemma 6.5. For any integers $m, n \geqslant 0$ and any integer $d>0$ the following statements are true:

(i) $\operatorname{Fr}_{m}^{<d}\left(-, X \times T^{n+1}\right)$ is a subpresheaf on Aff Sm/k of the presheaf $\operatorname{Fr}_{m}(-, X \times$ $\left.T^{n+1}\right)$;

(ii) the increasing filtration of the presheaf $\left.\operatorname{Fr}_{m}\left(-, X \times T^{n+1}\right)\right|_{A f f S m / k}$ by subpresheaves $\operatorname{Fr}_{m}^{<d}\left(-, X \times T^{n+1}\right)$ is exhausting;

(iii) $\left(\operatorname{Fr}_{m}^{q f}\right)^{<d}\left(-, X \times T^{n+1}\right)$ is a subpresheaf on AffSm/k of the presheaf $\operatorname{Fr}_{m}^{q f}\left(-, X \times T^{n+1}\right)$;

(iv) the increasing filtration of the presheaf $\left.\operatorname{Fr}_{m}^{q f}\left(-, X \times T^{n+1}\right)\right|_{A f f S m / k}$ by subpresheaves $\left(\mathrm{Fr}_{m}^{q f}\right)^{<d}\left(-, X \times T^{n+1}\right)$ is exhausting.

Definition 6.6. For an affine $k$-smooth $U$ we define $\mathbb{Z F}_{m}^{<d}\left(U, X \times T^{n+1}\right)$ as

$$
\begin{array}{r}
\mathbb{Z}\left[\operatorname{Fr}_{m}^{<d}\left(U, X \times T^{n+1}\right)\right] /\left\langle\left(Z_{1} \sqcup Z_{2}, W, \varphi ; g\right)-\left(Z_{1}, W_{2},\left.\varphi\right|_{W_{2}} ;\left.g\right|_{W_{2}}\right)-\right. \\
\left.-\left(Z_{2}, W_{1},\left.\varphi\right|_{W_{1}} ;\left.g\right|_{W_{1}}\right)\right\rangle,
\end{array}
$$


where $W_{i}=W-Z_{i}$ for $i=1,2$. By Lemma 6.5 the assignment $U \mapsto \mathbb{Z F}_{m}^{<d}(U, X \times$ $T^{n+1}$ ) is a presheaf on $\mathrm{AffSm} / \mathrm{k}$.

Likewise, for an affine $k$-smooth $U$ define $\left(\mathbb{Z F}_{m}^{q f}\right)^{<d}\left(U, X \times T^{n+1}\right)$ as

$$
\begin{aligned}
\mathbb{Z}\left[\left(\operatorname{Fr}_{m}^{q f}\right)^{<d}\left(U, X \times T^{n+1}\right)\right] /\left\langle\left( Z_{1}\right.\right. & \left.\sqcup Z_{2}, W, \varphi ; g\right)- \\
& \left.-\left(Z_{1}, W_{2},\left.\varphi\right|_{W_{2}} ;\left.g\right|_{W_{2}}\right)-\left(Z_{2}, W_{1},\left.\varphi\right|_{W_{1}} ;\left.g\right|_{W_{1}}\right)\right\rangle,
\end{aligned}
$$

where $W_{i}=W-Z_{i}$ for $i=1,2$. By Lemma 6.5 the assignment $U \mapsto\left(\mathbb{Z F}_{m}^{q f}\right)^{<d}(U, X \times$ $\left.T^{n+1}\right)$ is a presheaf on $\mathrm{AffSm} / \mathrm{k}$.

Recall that $\mathbb{Z F}_{m}\left(-, X \times T^{n+1}\right)$ is a presheaf on $S m / k$ (see Definition 2.3) given by

$\mathbb{Z}\left[\operatorname{Fr}_{m}\left(U, X \times T^{n+1}\right)\right] /\left\langle\left(Z_{1} \sqcup Z_{2}, W, \varphi ; g\right)-\left(Z_{1}, W_{2},\left.\varphi\right|_{W_{2}} ;\left.g\right|_{W_{2}}\right)-\left(Z_{2}, W_{1},\left.\varphi\right|_{W_{1}} ;\left.g\right|_{W_{1}}\right)\right\rangle$, where $W_{i}=W-Z_{i}$ for $i=1,2$. In turn, $\mathbb{Z F}_{m}^{q f}\left(-, X \times T^{n+1}\right)$ is a presheaf on $S m / k$ (see Definition 3.5) given by

$\mathbb{Z}\left[\operatorname{Fr}_{m}^{q f}\left(U, X \times T^{n+1}\right)\right] /\left\langle\left(Z_{1} \sqcup Z_{2}, W, \varphi ; g\right)-\left(Z_{1}, W_{2},\left.\varphi\right|_{W_{2}} ;\left.g\right|_{W_{2}}\right)-\left(Z_{2}, W_{1},\left.\varphi\right|_{W_{1}} ;\left.g\right|_{W_{1}}\right)\right\rangle$, where $W_{i}=W-Z_{i}$ for $i=1,2$.

For any positive integers $d<d^{\prime}$ the inclusion $\operatorname{Fr}_{m}^{<d}\left(-, X \times T^{n+1}\right) \subset \operatorname{Fr}_{m}^{<d}(-, X \times$ $\left.T^{n+1}\right)$ induces a morphism of presheaves of Abelian groups on $\mathrm{AffSm} / \mathrm{k}$

$$
\mathbb{Z F}_{m}^{<d}\left(U, X \times T^{n+1}\right) \rightarrow \mathbb{Z F}_{m}^{<d^{\prime}}\left(U, X \times T^{n+1}\right) .
$$

Likewise, for any positive integers $d<d^{\prime}$ the inclusion $\left(\operatorname{Fr}_{m}^{q f}\right)^{<d}\left(-, X \times T^{n+1}\right) \subset$ $\left(\operatorname{Fr}_{m}^{q f}\right)^{<d^{\prime}}\left(-, X \times T^{n+1}\right)$ induces a morphism of presheaves of Abelian groups on Aff Sm/k

$$
\left(\mathbb{Z} \mathrm{F}_{m}^{q f}\right)^{<d}\left(U, X \times T^{n+1}\right) \rightarrow\left(\mathbb{Z F}_{m}^{q f}\right)^{<d^{\prime}}\left(U, X \times T^{n+1}\right) .
$$

The next statement is a consequence of Lemma 6.5.

Corollary 6.7. One has two equalities of presheaves on Aff $\mathrm{Sm} / \mathrm{k}$

$$
\operatorname{colim}_{d} \mathbb{Z F}_{m}^{<d}\left(-, X \times T^{n+1}\right)=\mathbb{Z F}_{m}\left(-, X \times T^{n+1}\right)
$$

and

$$
\operatorname{colim}_{d}\left(\mathbb{Z F}_{m}^{q f}\right)^{<d}\left(-, X \times T^{n+1}\right)=\mathbb{Z F}_{m}^{q f}\left(-, X \times T^{n+1}\right) .
$$

Proof. This is straightforward.

\section{Moving LEMMA}

Lemma 7.1. ${ }^{1}$ Let $L$ be a field and let $F \in L\left[x_{1}, \ldots x_{r+1}\right]$ be a nonzero polynomial such that $\operatorname{deg} F \leqslant(d-1)$. Then the polynomials $F\left(t^{d^{r}}, t^{d^{r-1}}, \ldots, t^{d}, t\right)$ and $F\left(t^{d}, \ldots, t^{d^{r-1}}, t^{d^{r}}, t\right)$ are both non-zero in $L[t]$. Moreover, for any non-zero $s \in L$ the polynomials $F\left(s t^{d^{r}}, s t^{d^{r-1}}, \ldots, s t^{d}, t\right)$ and $F\left(s t^{d}, \ldots, s t^{d^{r-1}}, s t^{d^{r}}, t\right)$ are both non-zero.

Proof. Let us prove that the first polynomial is non-zero. If $F=\sum_{I=\left(i_{1}, \ldots, i_{r+1}\right)} a_{I} x^{I}$ then

$$
F\left(t^{d^{r}}, t^{d^{r-1}}, \ldots, t^{r}, t\right)=\sum a_{I} t^{i_{1} d^{r}+\ldots+i_{n} r+\ldots+i_{n+1}}
$$

\footnotetext{
${ }^{1}$ We thank A. Ananyevskiy for suggesting Lemma 7.1 in its present form.
} 
Let us check that if $I$ and $J$ are two different multi-indices, then $I \cdot\left(d^{r}, \ldots, d, 1\right) \neq$ $J \cdot\left(d^{r}, \ldots, d, 1\right)$. Indeed, if these are equal, then

$$
d^{r}\left(i_{1}-j_{1}\right)+\cdots+d\left(i_{n}-j_{n}\right)+\left(i_{n+1}-j_{n+1}\right)=0 .
$$

It follows that $i_{n+1}-j_{n+1}$ is divisible by $d$, but $\left|i_{n+1}-j_{n+1}\right| \leqslant d-1$, hence $i_{n+1}=j_{n+1}$. Then $\left(i_{n}-j_{n}\right)$ is divisible by $d$, hence zero and $\left(i_{1}-j_{1}\right)$ is zero by induction. Thus all powers of $t$ in the sum (7.1) are distinct. So if some $a_{I} \neq 0$ then the right hand side of (7.1) is nonzero. The second polynomial is obtained from the first one by permuting powers of $t$. Thus it is non-zero. For any multi-index $I$ the coefficient at $t^{i_{1} d^{r}+\ldots+i_{n} r+\ldots+i_{n+1}}$ in the polynomial $F\left(s t^{d^{r}}, s t^{d^{r-1}}, \ldots, s t^{d}, t\right)$ is obtained from $a_{I}$ by multiplying a power of $s$. We see that the polynomial $F\left(s t^{d^{r}}, s t^{d^{r-1}}, \ldots, s t^{d}, t\right)$ is non-zero, and hence so is $F\left(s t^{d}, \ldots, s t^{d^{r-1}}, s t^{d^{r}}, t\right)$.

Let $U \in S m / k$ be an affine variety and let $c=(Z, W, \varphi) \in \operatorname{Fr}_{m}\left(U, X \times T^{n+1} ; g\right)$ be a framed correspondence. Let $d>0$ be an integer. Set

$$
t_{d}(c)=\left(Z, W, \varphi_{1}-\varphi_{m+n+1}^{d}, \varphi_{2}-\varphi_{m+n+1}^{d^{2}}, \ldots, \varphi_{m+n}-\varphi_{m+n+1}^{d^{m+n}}, \varphi_{m+n+1} ; g\right) .
$$

We note that $Z\left(\varphi_{1}-\varphi_{m+n+1}^{d}, \varphi_{2}-\varphi_{m+n+1}^{d^{2}}, \ldots, \varphi_{m+n}-\varphi_{m+n+1}^{d^{m+n}}, \varphi_{m+n+1}\right)=$ $Z\left(\varphi_{1}, \ldots, \varphi_{m+n}, \varphi_{m+n+1}\right)$ in $W$. Thus the tuple $t_{d}(c)$ is an element in $\operatorname{Fr}_{n}(U, X \times$ $T^{n+1}$ ) such that its support $Z$ is the same with that of $c$. If $\tau$ is a variable, we put

$$
\begin{aligned}
& h^{d}(c)= \\
& \left(Z \times \mathbb{A}^{1}, W \times \mathbb{A}^{1}, \varphi_{1}-\tau \varphi_{m+n+1}^{d}, \varphi_{2}-\tau \varphi_{m+n+1}^{d^{2}}, \ldots, \varphi_{m+n}-\tau \varphi_{m+n+1}^{d^{m+n}}, \varphi_{m+n+1} ; g\right) .
\end{aligned}
$$

Note that $Z\left(\varphi_{1}-\tau \varphi_{m+n+1}^{d}, \ldots, \varphi_{m+n}-\tau \varphi_{m+n+1}^{d^{m+n}}, \varphi_{m+n+1}\right)=Z \times \mathbb{A}^{1}$ in $W \times \mathbb{A}^{1}$. Thus the tuple $h^{d}(c)$ is an element in $\operatorname{Fr}_{n}\left(U \times \mathbb{A}^{1}, X \times T^{n+1}\right)$ whose support equals $Z \times \mathbb{A}^{1}$.

Remark 7.2. Let $c=(Z, W, \varphi ; g) \in \operatorname{Fr}_{m}\left(U, X \times T^{n+1}\right)$ and let $\pi: W \rightarrow U$ be the composite map $U \stackrel{p r_{U}}{\longleftarrow} U \times \mathbb{A}^{m} \leftarrow W$. Then the map $(\varphi, \pi): W \rightarrow \mathbb{A}^{m+n+1} \times U$ is quasi-finite over $0 \times U$. Hence it is quasi-finite over some Zariski open neighborhood $V$ of $0 \times U$. Then $W^{\prime}=(\varphi, \pi)^{-1}(V)$ is a Zariski open neighborhood of $Z$ in $W$. Replacing $W$ by $W^{\prime}$ if necessary, we may and shall always assume in what follows that $(\varphi, \pi): W \rightarrow \mathbb{A}^{m+n+1} \times U$ is quasi-finite.

Lemma 7.3. If $U \in A f f S m / k$ then the following statements are true:

(i) if $c \in \operatorname{Fr}_{m}^{<d}\left(U, X \times T^{n+1}\right)$, then $t_{d}(c) \in \operatorname{Fr}_{m}^{q f}\left(U, X \times T^{n+1}\right)$;

(ii) if $c \in\left(\operatorname{Fr}_{m}^{q f}\right)^{<d}\left(U, X \times T^{n+1}\right)$, then $h^{d}(c) \in \operatorname{Fr}_{m}^{q f}\left(U \times \mathbb{A}^{1}, X \times T^{n+1}\right)$.

Proof. Prove the first assertion. Let $c=(Z, W, \varphi ; g) \in \operatorname{Fr}_{m}\left(U, X \times T^{n+1}\right) \in$ $\operatorname{Fr}_{m}^{<d}\left(U, X \times T^{n+1}\right)$. Let $F_{1}, \ldots F_{r} \in k\left[\mathbb{A}_{U}^{m+n+1}\right]$ be a $c$-defining set with $\operatorname{deg} F_{i}<d$ for all $i=1, \ldots, r$. We must check that $t_{d}(c)$ is in $\operatorname{Fr}_{m}^{q f}\left(U, X \times T^{n+1}\right)$. So, take

$$
Y=Z\left(\varphi_{1}-\varphi_{m+n+1}^{d}, \varphi_{2}-\varphi_{m+n+1}^{d^{2}}, \ldots, \varphi_{m+n}-\varphi_{m+n+1}^{d^{m+n}}\right) \subset W .
$$

Let $\pi: W \rightarrow U$ be the composite map $U \stackrel{p r_{U}}{\longleftarrow} U \times \mathbb{A}^{m} \leftarrow W$ as in Remark 7.2. We must check that $\left.\pi\right|_{Y}: Y \rightarrow U$ is quasi-finite. So we must check that for any point $u \in U$ the fiber $Y(u)$ of $Y$ over $u$ is finite. Let $\theta: \mathbb{A}^{1} \rightarrow \mathbb{A}^{m+n+1}$ be a morphism taking a point $t$ to the point $\left(t^{d}, t^{d^{2}}, \ldots, t^{d^{m+n}}, t\right)$. It is a closed embedding with the image $C=\theta\left(\mathbb{A}^{1}\right)$. 
By Remark 7.2 the morphism $\psi=(\varphi, \pi): W \rightarrow \mathbb{A}^{m+n+1} \times U$ is quasi-finite. Given a point $u \in U$ there is a polynomial $F$ from the $c$-defining set such that $F(-, u)$ is non-zero and its vanishing locus $Z(F(-, u))$ in $\mathbb{A}_{u}^{m+n+1}$ contains $\psi(W(u))$. Clearly, $Y(u)$ is contained in the set

$$
\psi^{-1}(Z(F(-, u)) \cap C) .
$$

The set $Z(F(-, u)) \cap C$ is in a bijection with the vanishing locus of the polynomial $F\left(t^{d}, t^{d^{2}}, \ldots, t^{d^{m+n}}, t\right)$ on the line $\mathbb{A}^{1}$ with the coordinate $t$. Thus by Lemma 7.1 the set $Z(F(-, u)) \cap C$ is finite, and hence so is $Y(u)$. The first assertion is proved.

Let us verify the second assertion. Let $c=(Z, W, \varphi ; g) \in\left(\operatorname{Fr}_{m}^{q f}\right)^{<d}\left(U, X \times T^{n+1}\right)$ and let $F_{1}, \ldots F_{r} \in k\left[\mathbb{A}_{U}^{m+n+1}\right]$ be a $c$-defining set with $\operatorname{deg} F_{i}<d$ for all $i=1, \ldots, r$. We must check that $h^{d}(c)$ is in $\operatorname{Fr}_{m}^{q f}\left(U \times \mathbb{A}^{1}, X \times T^{n+1}\right)$. So, take

$$
Y_{\tau}=Z\left(\varphi_{1}-\tau \varphi_{m+n+1}^{d}, \varphi_{2}-\tau \varphi_{m+n+1}^{d^{2}}, \ldots, \varphi_{m+n}-\tau \varphi_{m+n+1}^{d^{m+n}}\right) \subset W \times \mathbb{A}^{1},
$$

where $\tau$ is the coordinate on the additional factor $\mathbb{A}^{1}$. Let $\pi: W \rightarrow U$ be as above in this proof. Consider the map $\Pi=\pi \times i d_{\mathbb{A}^{1}}: W \times \mathbb{A}^{1} \rightarrow U \times \mathbb{A}^{1}$. We must check that $\left.\Pi\right|_{Y_{\tau}}: Y_{\tau} \rightarrow U \times \mathbb{A}^{1}$ is quasi-finite. So we must check that for any point $v \in U \times \mathbb{A}^{1}$ the fiber $Y_{\tau}(v)$ of $Y_{\tau}$ over $v$ is finite. Replacing the base field $k$ by its algebraic closure $\bar{k}$, we may assume that any point $v \in U \times \mathbb{A}^{1}$ is of the form $(u, a)$ with $a \in \bar{k}$. So, we must check that for any point $(u, a) \in U \times \mathbb{A}^{1}$ the fiber $Y_{a}(u)$ of $Y_{\tau}$ over $(u, a)$ is finite.

Given $0 \neq a \in \bar{k}$, let $\theta_{a}: \mathbb{A}^{1} \rightarrow \mathbb{A}^{m+n+1}$ be a morphism taking a point $t$ to the point $\left(a t^{d}, a t^{d^{2}}, \ldots, a t^{d^{m+n}}, t\right)$. It is a closed embedding with the image $C_{a}=\theta_{a}\left(\mathbb{A}^{1}\right)$. For $a=0$ let $\theta_{0}: \mathbb{A}^{1} \rightarrow \mathbb{A}^{m+n+1}$ be the morphism taking a point $t$ to $(0,0, \ldots, 0, t)$. It is a closed embedding with the image $C_{0}=\theta_{0}\left(\mathbb{A}^{1}\right)$. It is the last coordinate line $\mathbb{A}_{m+n+1}^{1}$ in $\mathbb{A}^{m+n+1}$.

By Remark 7.2 the morphism $\psi=(\varphi, \pi): W \rightarrow \mathbb{A}^{m+n+1} \times U$ is quasi-finite. Given a point $u \in U$, there is a polynomial $F$ from the $c$-defining set such that $F(-, u)$ is non-zero and its vanishing locus $Z(F(-, u))$ in $\mathbb{A}_{u}^{m+n+1}$ contains $\psi(W(u))$. For a given $0 \neq a \in \bar{k}$ the set $Y_{a}(u)$ is contained in the set

$$
\psi^{-1}\left(Z(F(-, u)) \cap C_{a}\right) .
$$

The set $\left.Z(F(-, u)) \cap C_{a}\right)$ is in a bijection with the vanishing locus of the polynomial $F\left(a t^{d}, a t^{d^{2}}, \ldots, a t^{d^{m+n}}, t\right)$ on the line $\mathbb{A}^{1}$ with the coordinate $t$. Thus by Lemma 7.1 the set $\left.Z(F(-, u)) \cap C_{a}\right)$ is finite in this case, and hence so is $Y_{a}(u)$.

For $a=0$, the set $Y_{0}(u)$ coincides with the closed subset $Z\left(\varphi_{1}, \ldots, \varphi_{m+n}\right)$ in $W$. It is quasi-finite over $U$, because $c=(Z, W, \varphi ; g) \in\left(\mathrm{Fr}_{m}^{q f}\right)^{<d}\left(U, X \times T^{n+1}\right)$. The second assertion is proved.

Lemma 7.3 implies that the assignment $c \mapsto t_{d}(c)$ gives a morphism of presheaves of pointed sets

$$
t_{d}: \operatorname{Fr}_{m}^{<d}\left(-, X \times T^{n+1}\right) \rightarrow \operatorname{Fr}_{m}^{q f}\left(-, X \times T^{n+1}\right)
$$

on $\operatorname{AffSm} / k$. It also implies that the assignment $c \mapsto h^{d}(c)$ gives a morphism of presheaves of pointed sets

$$
h_{d}: \operatorname{Fr}_{m}^{<d}\left(-, X \times T^{n+1}\right) \rightarrow \operatorname{Fr}_{m}^{q f}\left(-\times \mathbb{A}^{1}, X \times T^{n+1}\right)
$$


on $\operatorname{AffSm} / k$. Finally, the assignment $c \mapsto h_{d}(c)$ gives a morphism of presheaves of pointed sets

$$
h_{d}^{q f}:\left(\operatorname{Fr}_{m}^{q f}\right)^{<d}\left(-, X \times T^{n+1}\right) \rightarrow \operatorname{Fr}_{m}^{q f}\left(-\times \mathbb{A}^{1}, X \times T^{n+1}\right)
$$

on $\operatorname{AffSm} / k$.

Consider a diagram

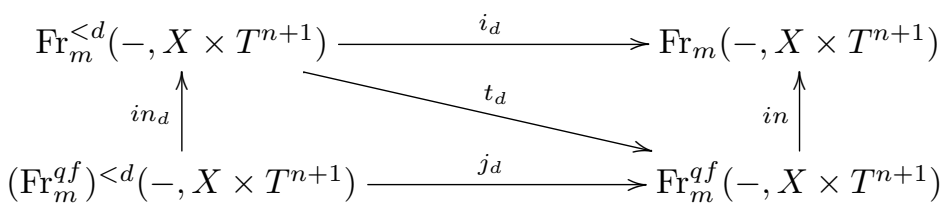

of presheaves of pointed sets on $A f f S m / k$. Lemma 7.3 shows that $h_{d}: \mathrm{Fr}_{m}^{<d}(-, X \times$ $\left.T^{n+1}\right) \rightarrow \underline{\operatorname{Hom}}\left(\mathbb{A}^{1}, \operatorname{Fr}_{m}\left(-, X \times T^{n+1}\right)\right)$ is an $\mathbb{A}^{1}$-homotopy between the morphisms $i n \circ t_{d}$ and $i_{d}$. It also shows that $h_{d}^{q f}:\left(\operatorname{Fr}_{m}^{q f}\right)^{<d}\left(-, X \times T^{n+1}\right) \rightarrow \underline{\operatorname{Hom}}\left(\mathbb{A}^{1}, \operatorname{Fr}_{m}^{q f}(-, X \times\right.$ $\left.\left.T^{n+1}\right)\right)$ is an $\mathbb{A}^{1}$-homotopy between the morphisms $t_{d} \circ i n_{d}$ and $j_{d}$.

Applying the free abelian group functor to the morphisms $t_{d}, i_{d}, j_{d}, i n_{d}$ and $i n$, we get certain morphisms between presheaves of abelian groups as well as two $\mathbb{A}^{1}$ homotopies (namely, $\mathbb{Z}\left[t_{d}\right], \mathbb{Z}\left[i_{d}\right], \mathbb{Z}\left[j_{d}\right], \mathbb{Z}\left[i n_{d}\right], \mathbb{Z}[i n], \mathbb{Z}\left[h_{d}\right]$ and $\mathbb{Z}\left[h_{d}^{q f}\right]$ ). Note that these morphisms and these two homotopies respect the additivity relations. Thus following Definition 6.6, we finally get morphisms $I_{d}, J_{d}, I n_{d}, I n$ and a morphism of presheaves

$$
T_{d}: \mathbb{Z F}_{m}^{<d}\left(-, X \times T^{n+1}\right) \rightarrow \mathbb{Z F}_{m}^{q f}\left(-, X \times T^{n+1}\right),
$$

and two $\mathbb{A}^{1}$-homotopies $H_{d}, H_{d}^{q f}$. In this way we get a diagram

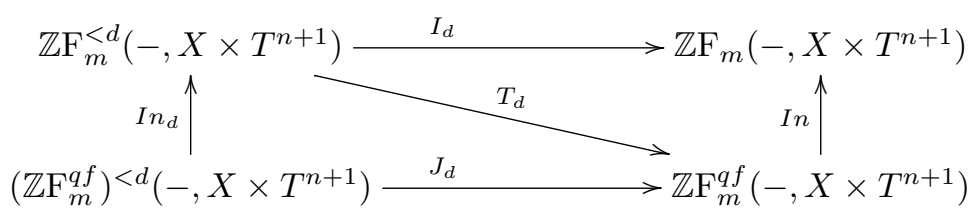

of presheaves of abelian groups on AffSm/k.

We document these arguments as follows.

Lemma 7.4. The $\mathbb{A}^{1}$-homotopy $h_{d}$ yields an $\mathbb{A}^{1}$-homotopy

$$
H_{d}: \mathbb{Z F}_{m}^{<d}\left(-, X \times T^{n+1}\right) \rightarrow \underline{\operatorname{Hom}}\left(\mathbb{A}^{1}, \mathbb{Z F}_{m}\left(-, X \times T^{n+1}\right)\right)
$$

between In $\circ T_{d}$ and $I_{d}$. The $\mathbb{A}^{1}$-homotopy $h_{d}^{q f}$ yields an $\mathbb{A}^{1}$-homotopy

$$
H_{d}^{q f}:\left(\mathbb{Z F}_{m}^{q f}\right)^{<d}\left(-, X \times T^{n+1}\right) \rightarrow \underline{\operatorname{Hom}}\left(\mathbb{A}^{1}, \mathbb{Z F}_{m}^{q f}\left(-, X \times T^{n+1}\right)\right)
$$

between $T_{d} \circ I n_{d}$ and $J_{d}$.

Proposition 7.5. For any integers $m, n \geqslant 0$ the morphism

$$
\text { In }: C_{*} \mathbb{Z} \mathrm{F}_{m}^{q f}\left(-, X \times T^{n+1}\right) \rightarrow C_{*} \mathbb{Z F}_{m}\left(-, X \times T^{n+1}\right)
$$

of complexes of presheaves of Abelian groups is a section-wise quasi-isomorphism on the category AffSm/k.

Proof of Proposition 7.5. The functor $C_{*}$ converts $\mathbb{A}^{1}$-homotopies to naive simplicial homotopies. Now the proposition follows from Lemma 7.4 and Corollary 6.7.

In more detail, for each integer $r \geqslant 0$ and each $k$-scheme $U \in A f f S m / k$, write $\mathbb{H}_{r}(U)$ for the $r$ th homology group of the complex $C_{*} \mathbb{Z} \mathrm{F}_{m}\left(U, X \times T^{n+1}\right)$ and 
write $\mathbb{H}_{r}^{q f}(U)$ for the $r$ th homology group of the complex $C_{*} \mathbb{Z F}_{m}^{q f}\left(U, X \times T^{n+1}\right)$. In addition, if $d>0$ is an integer, then write $\mathbb{H}_{r}^{(<d)}(U)$ for the $r$ th homology group of $C_{*} \mathbb{Z F}_{m}^{<d}\left(U, X \times T^{n+1}\right)$ and $\left(\mathbb{H}^{q f}\right)_{r}^{(<d)}(U)$ for the $r$ th homology group of $C_{*}\left(\mathbb{Z} \mathrm{F}_{m}^{q f}\right)^{<d}\left(U, X \times T^{n+1}\right)$. We choose the symbol $\mathbb{H}$ to avoid any confusion with $\mathbb{A}^{1}$-homotopies from Lemma 7.4. First, we have a commutative diagram of groups

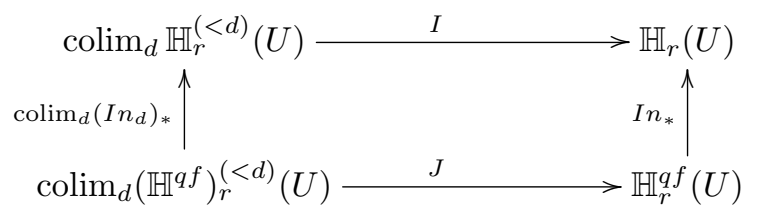

in which the arrows $I$ and $J$ are isomorphisms by Lemma 6.5. Second, for each integer $d>0$ we have a commutative diagram of groups

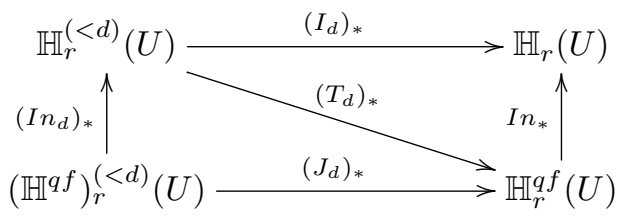

Since the arrows $I$ and $J$ from the commutative diagram (7.2) are isomorphisms and the upper (respectively, lower) triangle of the diagram (7.3) is commutative, it follows that $I n_{*}$ is surjective (respectively, injective). We see that $I n_{*}$ is an isomorphism. The proposition is proved.

We are now in a position to prove Theorem 1.5

Proof of Theorem 1.5. By Definitions 3.6 and 2.3 the complexes $C_{*} \mathbb{Z F}^{q f}(-, X \times$ $\left.T^{n+1}\right)$ ) and $\left.C_{*} \mathbb{Z F}\left(-, X \times T^{n+1}\right)\right)$ are the colimits of complexes $C_{*} \mathbb{Z F}_{m}^{q f}(-, X \times$ $\left.\left.T^{n+1}\right)\right)$ and $\left.C_{*} \mathbb{Z F}_{m}\left(-, X \times T^{n+1}\right)\right)$ over the suspension morphisms $\Sigma$. The morphisms In commute with the suspension morphisms $\Sigma$, i.e. $\Sigma \circ$ In $=$ In $\circ \Sigma$ : $C_{*} \mathbb{Z F}_{m}^{q f}\left(-, X \times T^{n+1}\right) \rightarrow C_{*} \mathbb{Z F}_{m+1}\left(-, X \times T^{n+1}\right)$. Proposition 7.5 now completes the proof.

\section{Proof of Theorem 1.1}

In this section Theorem 1.1 is proven, which is the main result of the paper. To prove the theorem, we need further definitions as well as Theorem 1.2 stated in the Introduction. Let $\Gamma^{\mathrm{op}}$ be the category of finite pointed sets and pointed maps. In what follows we shall identify $\Gamma^{\mathrm{op}}$ with a full subcategory of $\operatorname{Fr}_{0}(k)$. The identification sends $(K, *) \in \Gamma^{\mathrm{op}}$ to the non-pointed scheme $\operatorname{Spec} k \sqcup \ldots \sqcup \operatorname{Spec} k$, where the coproduct is indexed by the set $K^{\prime}=K \backslash *$. In turn, $\operatorname{Fr}_{0}(k)$ is a full subcategory of $\operatorname{SmOp}\left(\operatorname{Fr}_{0}(k)\right)$. Thus $\Gamma^{\mathrm{op}}$ is a full subcategory of $\operatorname{SmOp}\left(\operatorname{Fr}_{0}(k)\right)$. For each object $(Y, Y-S)$ in $\operatorname{SmOp}\left(\operatorname{Fr}_{0}(k)\right)$ and each finite pointed set $(K, *)$, we write $(Y, Y-S) \otimes K$ to denote $\left(Y \times K^{\prime},(Y-S) \times K^{\prime}\right)$ in $S m O p\left(\operatorname{Fr}_{0}(k)\right)$. In particular, if $Y \in \operatorname{Fr}_{0}(k)$, then $Y \otimes K=Y \times K^{\prime}=Y \sqcup \ldots \sqcup Y$ with the coproduct indexed by the elements of $K^{\prime}$. This notation is consistent with that in [GP1, Section 5].

Following Notation 2.8, let us define several $\Gamma$-spaces. Namely, if $U, X \in \operatorname{Fr}_{0}(k)$ and $m \geqslant 0$ is an integer, consider the following $\Gamma$-spaces:

$$
(K, *) \mapsto \operatorname{Fr}_{n}\left(U,\left(X \times T^{m}\right) \otimes K\right)
$$




$$
\begin{gathered}
(K, *) \mapsto \mathbb{Z F}_{n}\left(U,\left(X \times T^{m}\right) \otimes K\right), \\
(K, *) \mapsto \mathrm{F}_{n}\left(U,\left(X \times T^{m}\right) \otimes K\right),
\end{gathered}
$$

where the right hand side pointed sets correspond to the values of the relevant functors at the pair $\left(X \times\left(\mathbb{A}^{1}, \mathbb{G}_{m}\right)^{\wedge m}\right) \otimes K=\left(X \times \mathbb{A}^{m}, X \times\left(\mathbb{A}^{m}-\{0\}\right)\right) \otimes K$. By Definition 2.4 the set $\mathrm{F}_{n}\left(U,\left(X \times T^{m}\right) \otimes K\right)-0_{n}$ is a free basis of the abelian group $\mathbb{Z} \mathrm{F}_{n}\left(U,\left(X \times T^{m}\right) \otimes K\right)$.

Also, consider $\Gamma$-spaces

$$
\begin{gathered}
(K, *) \mapsto \mathbb{Z F r}_{*}\left(U,\left(X \times T^{n}\right) \otimes K\right), \\
(K, *) \mapsto \mathbb{Z F}_{*}\left(U,\left(X \times T^{n}\right) \otimes K\right) .
\end{gathered}
$$

The associated presheaves of Segal $S^{1}$-spectra will be denoted by $\mathbb{Z} \operatorname{Fr}_{*}^{S^{1}}\left(X \times T^{n}\right)$ and $\operatorname{EM}\left(\mathbb{Z F}_{*}\left(-, X \times T^{n}\right)\right)$, respectively. Thus,

$$
\mathbb{Z} \mathrm{Fr}_{*}^{S^{1}}\left(X \times T^{n}\right)=\left(\mathbb{Z F r}_{*}\left(-, X \times T^{n}\right), \mathbb{Z} \mathrm{Fr}_{*}\left(-,\left(X \times T^{n}\right) \otimes S^{1}\right), \ldots\right) .
$$

and

$$
E M\left(\mathbb{Z F}_{*}\left(-, X \times T^{n}\right)\right)=\left(\mathbb{Z F}_{*}\left(-, X \times T^{n}\right), \mathbb{Z F}_{*}\left(-,\left(X \times T^{n}\right) \otimes S^{1}\right), \ldots\right) .
$$

The equality $\mathbb{Z F}_{*}\left(-,\left(X \sqcup X^{\prime}\right) \times T^{n}\right)=\mathbb{Z F}_{*}\left(-, X \times T^{n}\right) \oplus \mathbb{Z F}_{*}\left(-, X^{\prime} \times T^{n}\right)$ implies that the $\Gamma$-space $(K, *) \mapsto \mathbb{Z F}_{*}\left(U,\left(X \times T^{n}\right) \otimes K\right)$ is fully determined by the abelian group $\mathbb{Z F}_{*}\left(U, X \times T^{n}\right)$. Hence $E M\left(\mathbb{Z F}_{*}\left(-, X \times T^{n}\right)\right)$ is the Eilenberg-Mac Lane spectrum for $\mathbb{Z} \mathrm{F}_{*}\left(-, X \times T^{n}\right)$.

The morphism of $\Gamma$-spaces $\left[(K, *) \mapsto \mathbb{Z F r}_{*}\left(-,\left(X \times T^{n}\right) \otimes K\right)\right] \rightarrow[(K, *) \mapsto$ $\left.\mathbb{Z} \mathrm{F}_{*}\left(-,\left(X \times T^{n}\right) \otimes K\right)\right]$ induces a morphism of framed $S^{1}$-spectra

$$
\lambda_{X \times T^{n}}: \mathbb{Z F r}_{*}^{S^{1}}\left(X \times T^{n}\right) \rightarrow E M\left(\mathbb{Z} \mathrm{F}_{*}\left(-, X \times T^{n}\right)\right) .
$$

Also, denote by $\mathbb{Z} M_{f r}\left(X \times T^{n}\right), X \in S m / k$, the Segal $S^{1}$-spectrum

$$
\left(C_{*} \mathbb{Z} \operatorname{Fr}\left(-, X \times T^{n}\right), C_{*} \mathbb{Z} \operatorname{Fr}\left(-,\left(X \times T^{n}\right) \otimes S^{1}\right), \ldots\right) .
$$

Let $L M_{f r}\left(X \times T^{n}\right)$ be the Segal $S^{1}$-spectrum

$$
\operatorname{EM}\left(\mathbb{Z F}\left(\Delta^{\bullet} \times-, X \times T^{n}\right)\right)=\left(\mathbb{Z F}\left(\Delta^{\bullet} \times-, X \times T^{n}\right), \mathbb{Z F}\left(\Delta \bullet \times-,\left(X \times T^{n}\right) \otimes S^{1}\right), \ldots\right) .
$$

The above arguments show that $L M_{f r}\left(X \times T^{n}\right)$ is the Eilenberg-Mac Lane spectrum associated with the complex $\mathbb{Z F}\left(\Delta^{\bullet} \times-, X \times T^{n}\right)$. The $\Gamma$-space morphism

$$
\left[(K, *) \mapsto \mathbb{Z} \operatorname{Fr}\left(\Delta^{\bullet} \times-,\left(X \times T^{n}\right) \otimes K\right)\right] \rightarrow\left[(K, *) \mapsto \mathbb{Z F}\left(\Delta^{\bullet} \times-,\left(X \times T^{n}\right) \otimes K\right)\right]
$$

induces a morphism of framed $S^{1}$-spectra

$$
l_{X \times T^{n}}: \mathbb{Z} M_{f r}\left(X \times T^{n}\right) \rightarrow L M_{f r}\left(X \times T^{n}\right) .
$$

Note that stable homotopy groups of $L M_{f r}\left(X \times T^{n}\right)=E M\left(\mathbb{Z F}\left(\Delta^{\bullet} \times-, X \times T^{n}\right)\right)$ are equal to homology groups of the complex $\mathbb{Z F}\left(\Delta^{\bullet} \times-, X \times T^{n}\right)$. By [Sch, $\S$ II.6.2] homotopy groups $\pi_{*}\left(\mathbb{Z} M_{f r}\left(X \times T^{n}\right)(U)\right)$ of the $S^{1}$-spectrum $\mathbb{Z} M_{f r}\left(X \times T^{n}\right)$ evaluated at $U \in \operatorname{Fr}_{0}(k)$ are homology groups $H_{*}\left(M_{f r}\left(X \times T^{n}\right)(U)\right)$ of $M_{f r}(X \times$ $\left.T^{n}\right)(U)$.

For the convenience of the reader we recall Theorem 1.2 proved in Appendix B. It computes, in particular, homology of the framed motive $M_{f_{r}}\left(X \times T^{n}\right)$ of the relative motivic sphere $X \times \mathbb{A}^{n} /\left(X \times\left(\mathbb{A}^{n}-\{0\}\right)\right)$. 
Theorem 1.2. For any integer $m \geqslant 0$, the natural morphism of framed $S^{1}$-spectra

$$
\lambda_{X \times T^{m}}: \mathbb{Z} \operatorname{Fr}_{*}^{S^{1}}\left(X \times T^{m}\right) \rightarrow E M\left(\mathbb{Z} \mathrm{F}_{*}\left(-, X \times T^{m}\right)\right)
$$

is a schemewise stable equivalence. Moreover, the natural morphism of framed $S^{1}$ spectra

$$
l_{X \times T^{m}}: \mathbb{Z} M_{f r}\left(X \times T^{m}\right) \rightarrow L M_{f r}\left(X \times T^{m}\right)
$$

is a schemewise stable equivalence. In particular, for any $U \in S m / k$ one has

$$
\pi_{*}\left(\mathbb{Z} M_{f r}\left(X \times T^{m}\right)(U)\right)=H_{*}\left(\mathbb{Z} \mathrm{F}\left(\Delta^{\bullet} \times U, X \times T^{m}\right)\right)=H_{*}\left(C_{*} \mathbb{Z} \mathrm{F}\left(U, X \times T^{m}\right)\right) .
$$

As above, we can define the following $\Gamma$-space:

$$
(K, *) \mapsto \operatorname{Fr}\left(\Delta^{\bullet} \times-,\left(X \times T^{m}\right) \otimes K\right) .
$$

The Segal $S^{1}$-spectrum associated to this $\Gamma$-space is denoted by $M_{f r}\left(X \times T^{m}\right)$. By construction,

$$
M_{f r}\left(X \times T^{m}\right)=\left(C_{*} \operatorname{Fr}\left(-, X \times T^{m}\right), C_{*} \operatorname{Fr}\left(-,\left(X \times T^{m}\right) \otimes S^{1}\right), \ldots\right) .
$$

We are now in a position to prove the remaining Theorem 1.1.

Proof of Theorem 1.1. By Theorem 1.3 the map of complexes of presheaves of Abelian groups

$$
C_{*} \mathbb{Z} \mathrm{F}\left(X \times T^{n} \times \mathbb{A}^{1}\right) / C_{*} \mathbb{Z} \mathrm{F}\left(X \times T^{n} \times \mathbb{G}_{m}\right) \rightarrow C_{*} \mathbb{Z} \mathrm{F}\left(X \times T^{n+1}\right)
$$

is a local quasi-isomorphism. The $S^{1}$-spectra $L M_{f r}\left(X \times T^{n} \times \mathbb{A}^{1}\right), L M_{f r}\left(X \times T^{n} \times\right.$ $\left.\mathbb{G}_{m}\right)$ and $L M_{f r}\left(X \times T^{n+1}\right)$ are the Eilenberg-Maclane $S^{1}$-spectra of the complexes $C_{*} \mathbb{Z} \mathrm{F}\left(X \times T^{n} \times \mathbb{A}^{1}\right), C_{*} \mathbb{Z} \mathrm{F}\left(X \times T^{n} \times \mathbb{G}_{m}\right)$ and $C_{*} \mathbb{Z} \mathrm{F}\left(X \times T^{n+1}\right)$ respectively. Thus the map

$$
L M_{f r}\left(X \times T^{n} \times \mathbb{A}^{1}\right) / L M_{f r}\left(X \times T^{n} \times \mathbb{G}_{m}\right) \rightarrow L M_{f r}\left(X \times T^{n+1}\right),
$$

induced by (8.4), is a local stable weak equivalence, and hence so is the map

$$
\mathbb{Z} M_{f r}\left(X \times T^{n} \times \mathbb{A}^{1}\right) / \mathbb{Z} M_{f r}\left(X \times T^{n} \times \mathbb{G}_{m}\right) \rightarrow \mathbb{Z} M_{f r}\left(X \times T^{n+1}\right)
$$

by Theorem 1.2. The $S^{1}$-spectra $M_{f r}\left(X \times T^{n} \times \mathbb{A}^{1}\right), M_{f r}\left(X \times T^{n} \times \mathbb{G}_{m}\right), M_{f r}(X \times$ $\left.T^{n+1}\right)$ are connected. Now the stable Whitehead theorem [Sch, II.6.30] implies the map

$$
M_{f r}\left(X \times T^{n} \times \mathbb{A}^{1}\right) / M_{f r}\left(X \times T^{n} \times \mathbb{G}_{m}\right) \rightarrow M_{f r}\left(X \times T^{n+1}\right)
$$

is a local stable weak equivalence. It follows that the sequence of $S^{1}$-spectra

$$
M_{f r}\left(X \times T^{n} \times \mathbb{G}_{m}\right) \rightarrow M_{f r}\left(X \times T^{n} \times \mathbb{A}^{1}\right) \rightarrow M_{f r}\left(X \times T^{n+1}\right)
$$

is locally a homotopy cofiber sequence. This proves the second assertion of the theorem.

Next, prove that for any $\ell \geqslant 1$ the canonical morphism

$$
M_{f r}\left(\operatorname{id}_{X} \times \alpha^{\wedge \ell}\right): M_{f r}\left(X \times\left(\mathbb{A}^{1} / / \mathbb{G}_{m}\right)^{\wedge \ell}\right) \rightarrow M_{f r}\left(X \times T^{\ell}\right)
$$

is a local stable weak equivalence. As above, this reduces to verifying that the canonical morphism of complexes of presheaves

$$
C_{*} \mathbb{Z} \mathrm{F}\left(\operatorname{id}_{X} \times \alpha^{\wedge \ell}\right): C_{*} \mathbb{Z} \mathrm{F}\left(X \times\left(\mathbb{A}^{1} / / \mathbb{G}_{m}\right)^{\wedge \ell}\right) \rightarrow C_{*} \mathbb{Z F}\left(X \times T^{\ell}\right)
$$

is locally a quasi-isomorphism. We verify this using induction by $\ell$. First, take $\ell=1$. Let $\mu: \mathbb{Z F}\left(\mathrm{id}_{X} \times \mathbb{A}^{1} / / \mathbb{G}_{m}\right) \rightarrow \mathbb{Z F}\left(X \times \mathbb{A}^{1}\right) / \mathbb{Z F}\left(X \times \mathbb{G}_{m}\right)$ be the canonical morphism. Then $C_{*}(\mu)$ is a sectionwise quasi-isomorphism. Also, $C_{*} \mathbb{Z F}\left(\operatorname{id}_{X} \times \alpha\right)=$ $\tau \circ C_{*}(\mu)$ with $\tau$ as in Theorem 1.3. Thus by Theorem $1.3 C_{*} \mathbb{Z F}\left(\operatorname{id}_{X} \times \alpha\right)$ is locally a 
quasi-isomorphism. We have verified the induction base case. To do the inductiion step from $\ell-1$ to $\ell$, note that

$$
C_{*} \mathbb{Z F}\left(\operatorname{id}_{X} \times \alpha^{\wedge \ell}\right)=C_{*} \mathbb{Z F}(\operatorname{id} \times \alpha) \circ C_{*} \mathbb{Z F}\left(\operatorname{id}_{X} \times \alpha^{\wedge(\ell-1)} \times \operatorname{id}_{\mathbb{A}^{1} / / \mathbb{G}_{m}}\right) .
$$

The map $C_{*} \mathbb{Z F}(\mathrm{id} \times \alpha)$ is locally a quasi-isomorphism by the case $\ell=1$. The morphism $C_{*} \mathbb{Z F}\left(\mathrm{id}_{X} \times \alpha^{\wedge(\ell-1)} \times \operatorname{id}_{\mathbb{A}^{1} / / \mathbb{G}_{m}}\right)$ is locally a quasi-isomorphism by the induction assumption. We see that $C_{*} \mathbb{Z} \mathrm{F}\left(\mathrm{id}_{X} \times \alpha^{\wedge \ell}\right)$ is locally a quasi-isomorphism, and hence $M_{f r}\left(\operatorname{id}_{X} \times \alpha^{\wedge \ell}\right)$ is a local stable weak equivalence for each integer $\ell \geqslant 1$.

It remains to prove that for each integer $\ell \geqslant 1$ the morphism (8.6) is a local level weak equivalence. By the Additivity Theorem of [GP1] and Lemma A.1 the $S^{1}$-spectrum $M_{f r}\left(X \times T^{\ell}\right)(U)$ with $U$ a local Henselian smooth scheme and $X$ any smooth scheme is a connected $\Omega$-spectrum. By the Additivity Theorem of [GP1] the $\Gamma$-space $K \rightarrow C_{*} \operatorname{Fr}\left(U,\left(X \times\left(\mathbb{A}^{1} / / \mathbb{G}_{m}\right)^{\wedge \ell}\right) \otimes K\right)$ is special. By Lemma A.1 the zeroth space $C_{*} \operatorname{Fr}\left(-, X \times\left(\mathbb{A}^{1} / / \mathbb{G}_{m}\right)^{\wedge \ell}\right)$ of the Segal $S^{1}$-spectrum $M_{f r}(X \times$ $\left.\left(\mathbb{A}^{1} / / \mathbb{G}_{m}\right)^{\wedge \ell}\right)(U)$ is connected. We see that the Segal $S^{1}$-spectrum $M_{f r}(X \times$ $\left.\left(\mathbb{A}^{1} / / \mathbb{G}_{m}\right)^{\wedge \ell}\right)(U)$ is a connected $\Omega$-spectrum. Since the morphism (8.6) is locally a stable weak equivalence between $\Omega$-spectra, it is a local level weak equivalence. The theorem is proved.

Corollary 8.1. Let $k$ be an infinite perfect field. For every $n \geqslant 0$, the natural morphism

$$
M_{f r}\left(X \times T^{n} \times\left(\mathbb{A}^{1} / / \mathbb{G}_{m}\right)\right) \rightarrow M_{f r}\left(X \times T^{n+1}\right)
$$

is locally a level weak equivalence of $S^{1}$-spectra.

Proof. Consider a commutative diagram

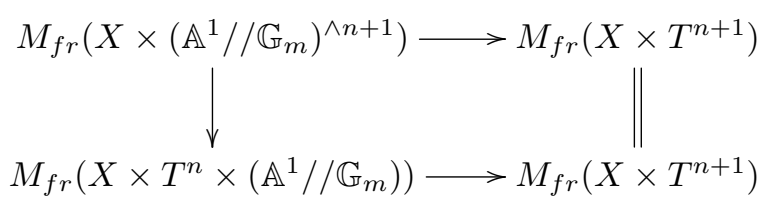

The left vertical and upper horizontal arrows are locally level weak equivalences of $S^{1}$-spectra by Theorem 1.1, and hence so is the lower horizontal arrow.

\section{Appendix A}

In this section we prove the following useful

Lemma A.1. For any $X \in S m / k$ and any $n>0$ the simplicial pointed presheaves $C_{*} \operatorname{Fr}\left(-, X \times\left(\mathbb{A}^{1} / / \mathbb{G}_{m}\right)^{\wedge n}\right)$ and $C_{*} \operatorname{Fr}\left(-, X \times T^{n}\right)$ are locally connected in the Nisnevich topology.

Proof. Firstly check the Nisnevich local connectivity of $C_{*} \operatorname{Fr}\left(-, X \times\left(\mathbb{A}^{1} / / \mathbb{G}_{m}\right)\right)$. Clearly, the map $\pi_{0}\left(C_{*} \operatorname{Fr}\left(-, X \times \mathbb{A}^{1}\right)\right) \rightarrow \pi_{0}\left(C_{*} \operatorname{Fr}\left(-, X \times\left(\mathbb{A}^{1} / / \mathbb{G}_{m}\right)\right)\right)$ is surjective. On the other hand the composite map of pointed sets

$$
\pi_{0}\left(C_{*} \operatorname{Fr}\left(-, X \times \mathbb{G}_{m}\right)\right) \rightarrow \pi_{0}\left(C_{*} \operatorname{Fr}\left(-, X \times \mathbb{A}^{1}\right)\right) \rightarrow \pi_{0}\left(C_{*} \operatorname{Fr}\left(-, X \times\left(\mathbb{A}^{1} / / \mathbb{G}_{m}\right)\right)\right)
$$

is constant, because it factors through the pointed set $\pi_{0}\left(C_{*} \operatorname{Fr}\left(-,\left(X \times \mathbb{G}_{m}\right) \otimes I\right)\right)=$ *. Thus it is sufficient to check that for any local essentially $k$-smooth Henselian $U$ the map

$$
\pi_{0}\left(C_{*} \operatorname{Fr}\left(U, X \times \mathbb{G}_{m}\right)\right) \rightarrow \pi_{0}\left(C_{*} \operatorname{Fr}\left(U, X \times \mathbb{A}^{1}\right)\right)
$$


is surjective. Take a framed correspondence

$$
c_{0}=\left(Z, W, \varphi ;(f, g): W \rightarrow X \times \mathbb{A}^{1}\right) \in \operatorname{Fr}_{n}\left(U, X \times \mathbb{A}^{1}\right)
$$

We may now assume that $W=\left(\mathbb{A}_{U}^{n}\right)_{Z}^{h}$ is the henselization of $\mathbb{A}_{U}^{n}$ at the closed subset $Z$. Since the scheme $\mathbb{A}_{U}^{n}$ is affine Noetherian, $\left(\mathbb{A}_{U}^{n}\right)_{Z}^{h}$ is an affine Noetherian scheme (see $[\mathrm{G}, 6.9])$. We want to find $h_{t} \in \operatorname{Fr}_{n}\left(\mathbb{A}_{U}^{1}, X \times \mathbb{A}^{1}\right)$ and $c_{1} \in \operatorname{Fr}_{n}\left(U, X \times \mathbb{G}_{m, k}\right)$ such that $h_{0}=c_{0}, h_{1}=j \circ c_{1}$, where $j: X \times \mathbb{G}_{m} \hookrightarrow X \times \mathbb{A}_{k}^{1}$ is the open embedding.

Construct now a required element $h_{t} \in \operatorname{Fr}_{n}\left(\mathbb{A}_{U}^{1}, X \times \mathbb{A}^{1}\right)$. To do that consider a closed subset $\tilde{Z}$ in $\mathbb{A}^{1} \times Z$ defined by the equation $t=\left.g\right|_{Z}$. Clearly, $\tilde{Z}$ is isomorphic to $Z$. Hence it is finite over $U$. Since the field $k$ is infinite and $\tilde{Z}$ is finite over $U$, there is a non-zero element $a \in k$ such that $(a \times Z) \cap \tilde{Z}=\emptyset$ in $\mathbb{A}^{1} \times Z$. We may assume that $a=1$. Put

$$
h_{t}=\left(\tilde{Z}, \mathbb{A}^{1} \times W, \varphi_{1}, \ldots, \varphi_{n}, g-t ; f: W \rightarrow X\right) \in \operatorname{Fr}_{n}\left(\mathbb{A}_{U}^{1}, X \times T\right) .
$$

Clearly, $h_{0}=b_{1}$ and $h_{1}=j \circ c_{1}$ for certain $c_{1} \in \operatorname{Fr}_{n}\left(U, X \times \mathbb{G}_{m, k}\right)$. Thus the arrow (A.1) is surjective and $C_{*} \operatorname{Fr}\left(-, X \times\left(\mathbb{A}^{1} / / \mathbb{G}_{m}\right)\right)$ is locally connected.

By induction, suppose $C_{*} \operatorname{Fr}\left(-, X \times\left(\mathbb{A}^{1} / / \mathbb{G}_{m}\right)^{\wedge n}\right)$ is locally connected. Then $C_{*} \operatorname{Fr}\left(-, X \times\left(\mathbb{A}^{1} / / \mathbb{G}_{m}\right)^{\wedge(n+1)}\right)$ is the realization of a simplicial space of the form

$$
[r] \mapsto C_{*} \operatorname{Fr}\left(-, Y_{r} \times\left(\mathbb{A}^{1} / / \mathbb{G}_{m}\right)^{\wedge n}\right)
$$

with $Y_{r} \in S m / k$. Since each $C_{*} \operatorname{Fr}\left(-, Y_{r} \times\left(\mathbb{A}^{1} / / \mathbb{G}_{m}\right)^{\wedge n}\right)$ is locally connected by the induction hypothesis, then $C_{*} \operatorname{Fr}\left(-, X \times\left(\mathbb{A}^{1} / / \mathbb{G}_{m}\right)^{\wedge(n+1)}\right)$ is locally connected as well.

Now let us prove the Nisnevich local connectivity of $C_{*} \operatorname{Fr}\left(-, X \times T^{n}\right)$. We give a proof for $n=1$. The general case is treated similarly. For any local essentially $k$ smooth Henselian $U$, consider $b_{0}=\left(Z, V, \varphi_{1}, \ldots, \varphi_{n}, \varphi_{n+1} ; f: V \rightarrow X\right) \in \operatorname{Fr}_{n}(U, X \times$ $T)$. It is sufficient to find a family of elements

$$
\left\{h_{t}^{(0)}, h_{t}^{(1)}, \ldots, h_{t}^{(r)}\right\} \subset \operatorname{Fr}_{n}\left(\mathbb{A}_{U}^{1}, X \times T\right)
$$

such that $h_{0}^{(0)}=b_{0}, h_{1}^{(i)}=h_{0}^{(i+1)}$ for $i=0, \ldots, r-1$ and $h_{1}^{(r)}=0_{n}$ is the empty framed correspondence. By Lemma 7.3 we may assume that $b_{1}:=h_{1}^{(0)}$ is in $\operatorname{Fr}_{n}^{q f}\left(\mathbb{A}_{U}^{1}, X \times T\right)$. Write $b_{1}$ as $b_{1}=\left(Z, W, \psi_{1}, \ldots, \psi_{n}, \psi_{n+1} ; g: W \rightarrow X\right)$. We may assume that $W=\left(\mathbb{A}_{U}^{n}\right)_{Z}^{h}$ is the henselization of $\mathbb{A}_{U}^{n}$ at the closed subset $Z$. Since $b_{1}$ is in $\operatorname{Fr}_{n}^{q f}\left(\mathbb{A}_{U}^{1}, X \times T\right)$ the closed subset $Y:=\left\{\psi_{1}=\psi_{2}=\ldots=\psi_{n}=0\right\}$ of $W$ is quasi-finite over $U$. The following lemma shows that we may assume $Y$ is finite over $U$.

Lemma A.2. There is an affine Zariski open $W^{0} \subset W$ such that the closed subset $Y^{0}:=W^{0} \cap Y$ is finite over $U, Z$ is contained in $W^{0}$, and $\left(Z, W^{0}, \psi_{1}, \ldots, \psi_{n}, \psi_{n+1} ; g\right)$ $=\left(Z, W, \psi_{1}, \ldots, \psi_{n}, \psi_{n+1} ; g\right)$.

Proof. Before proving the lemma note that its first two assertions yield the last one. To prove the lemma, consider a closed subset $Y^{\prime}$ of $Y$ which is the union of all connected components $Y_{i}^{\prime}$ of $Y$ having non-empty intersection with $Z$. Let $Y^{\prime \prime}$ be the union of all other connected components of $Y$. Clearly, $Y=Y^{\prime} \sqcup Y^{\prime \prime}$. Put $W^{0}=W-Y^{\prime \prime}$ and $Y^{0}=W^{0} \cap Y$. Then $Y^{\prime}=Y^{0}$. It remains to check that $Y^{\prime}$ is finite over the local henselian scheme $U$. It is sufficient to check that each $Y_{i}^{\prime}$ is finite over $U$. Put $S_{i}=Y_{i}^{\prime} \cap Z$. Then $S_{i}$ is a non-empty closed subset of $Z$. So $S_{i}$ is non-empty and finite over $U$. Thus the fibre $S_{i, u}$ over the closed point $u \in U$ 
is non-empty, and hence so is the fibre $Y_{i, u}^{\prime}$ of $Y_{i}^{\prime}$ over $u$. Now [Mi, Theorem I.4.2] implies finiteness of $Y_{i}^{\prime}$ over $U$. Lemma A.2 is proved.

Construct now an element $H_{t} \in \operatorname{Fr}_{n}\left(\mathbb{A}_{U}^{1}, X \times T\right)$ such that $H_{0}=b_{1}$ and $H_{1}=0_{n}$. Consider a closed subset $\tilde{Y}$ in $\mathbb{A}^{1} \times Y$ defined by the equation $t=\left.\psi_{n+1}\right|_{Y}$. Clearly, $\tilde{Y}$ is isomorphic to $Y$. Hence it is finite over $U$. Since the field $k$ is infinite and $\tilde{Y}$ is finite over $U$, there is a non-zero element $a \in k$ such that $(a \times Y) \cap \tilde{Y}=\emptyset$ in $\mathbb{A}^{1} \times Y$. We may assume that $a=1$. Put

$$
H_{t}=\left(\tilde{Y}, \mathbb{A}^{1} \times W, \psi_{1}, \ldots, \psi_{n}, \psi_{n+1}-t ; g: W \rightarrow X\right) \in \operatorname{Fr}_{n}\left(\mathbb{A}_{U}^{1}, X \times T\right) .
$$

Clearly, $H_{0}=b_{1}$ and $H=0_{n}$. Thus $C_{*} \operatorname{Fr}(-, X \times T)$ is locally connected. The same is true for $C_{*} \operatorname{Fr}\left(-, X \times T^{n}\right)$. This proves Lemma A.1.

\section{Appendix B}

The main goal of this section is to prove Theorem 1.2. It will be proved at the end of the section.

Let $\mathbb{S}$ be the sphere $S^{1}$-spectrum. Let $* \subset \mathbb{S}$ be its trivial $S^{1}$-subspectrum corresponding to the basepoint. Let $\mathcal{A}$ be a pointed set with a distinguished point *. Denote by $\mathbb{S}_{\mathcal{A}}$ the $S^{1}$-spectrum $\prod_{(\mathcal{A}-*)} \mathbb{S}$. Let $\mathbb{S}_{\mathcal{A}}^{\prime}$ be the $S^{1}$-subspectrum $\vee_{(\mathcal{A}-*)} \mathbb{S}$ in $\mathbb{S}_{\mathcal{A}}$.

Given a finite pointed subset $A \subset \mathcal{A}$, let $\mathbb{S}_{A} \subset \mathbb{S}_{\mathcal{A}}$ be an $S^{1}$-subspectrum of the form $\prod_{(A-*)} E_{a}$, where $E_{a}=\mathbb{S}$ if $a \in A-*$ and $E_{a}=*$ if $a \in \mathcal{A}-A$. If $a \in A-*$ we shall write $\mathbb{S}_{a}$ to denote $\mathbb{S}_{\{a, *\}}$, where $\{a, *\} \subset A$ is the two elements subset of $A$. Let $\mathbb{S}_{A}^{\prime} \subset \mathbb{S}_{A}$ be the $S^{1}$-subspectrum $\vee_{a \in(A-*)} \mathbb{S}_{a}$ in $\mathbb{S}_{A}$. Clearly, the inclusion $\mathbb{S}_{A}^{\prime} \subset \mathbb{S}_{A}$ is a stable equivalence of $S^{1}$-spectra. Set $\mathbb{S}_{\mathcal{A}}^{f}:=\cup_{A \subset \mathcal{A}} \mathbb{S}_{A} \subset \mathbb{S}_{\mathcal{A}}$, where the union is taken over the set of all finite pointed subsets $A$ of the pointed set $\mathcal{A}$.

The following lemma is straightforward and the proof is left to the reader.

Lemma B.1. Let $\mathcal{A}$ be a pointed set and I be a set such that for any $i \in I$ there is a finite pointed subset $A(i) \subset \mathcal{A}$. We have two $S^{1}$-subspectra $\cup_{i \in I} \mathbb{S}_{A(i)}^{\prime}, \cup_{i \in I} \mathbb{S}_{A(i)}$ of the spectrum $\mathbb{S}_{\mathcal{A}}^{f}$. Suppose $\cup_{i \in I} A(i)=\mathcal{A}$. Then $\vee_{(\mathcal{A}-*)} \mathbb{S}_{a}=\cup_{i \in I} \mathbb{S}_{A(i)}^{\prime}$ and the inclusion

$$
\vee_{(\mathcal{A}-*)} \mathbb{S}_{a}=\cup_{i \in I} \mathbb{S}_{A(i)}^{\prime} \hookrightarrow \cup_{i \in I} \mathbb{S}_{A(i)}
$$

is a stable equivalence of $S^{1}$-spectra.

An application of this lemma is given below in this section. For a finite pointed set $(K, *)$ consider a set $\operatorname{Map}_{\bullet}^{f}(\mathcal{A}, K)$ of those maps $\rho$ of pointed sets such that the set $\rho^{-1}(K-*)$ is finite. Consider a $\Gamma$-space $\Gamma_{\mathcal{A}}^{f}$ defined by $\Gamma_{\mathcal{A}}^{f}(K, *)=\operatorname{Map}{ }_{\bullet}^{f}(\mathcal{A}, K)$. For a finite pointed subset $A \subset \mathcal{A}$ consider a subset $\operatorname{Map}_{\bullet}^{A}(\mathcal{A}, K) \subset \operatorname{Map}_{\bullet}^{f}(\mathcal{A}, K)$ consisting of all maps $\rho$ such that $\rho^{-1}(K-*) \subset A$. Consider a $\Gamma$-space $\Gamma_{A}$ defined by $\Gamma_{A}(K, *)=\operatorname{Map} \bullet_{\bullet}^{A}(\mathcal{A}, K)$.

Clearly, for any inclusion of finite pointed subsets $A^{\prime} \subset A$ of $\mathcal{A}$ one has inclusions $\operatorname{Map}_{\bullet}^{A^{\prime}}(\mathcal{A}, K) \subset \operatorname{Map}_{\bullet}^{A}(\mathcal{A}, K)$ and $\Gamma_{A^{\prime}} \subset \Gamma_{A}$. Moreover, one has

$$
\cup_{A \subset \mathcal{A}} M a p_{\bullet}^{A}(\mathcal{A}, K)=\operatorname{Map} p_{\bullet}^{f}(\mathcal{A}, K) \text { and } \cup_{A \subset \mathcal{A}} \Gamma_{A}=\Gamma_{\mathcal{A}}^{f},
$$

where the union is taken over the set of all pointed finite subsets $A$ in the pointed set $\mathcal{A}$. 
Let $A \subset \mathcal{A}$ be a finite pointed subset. For any element $a \in A-* \operatorname{set} \operatorname{Map}_{\bullet}^{a}(\mathcal{A}, K)=$ $\operatorname{Map}_{\bullet}^{a \sqcup *}(\mathcal{A}, K)$, where $a \sqcup *$ stands for the two elements pointed subset of $\mathcal{A}$. Set $\Gamma_{a}=\Gamma_{a \sqcup *}$. That is $\Gamma_{a}(K, *)=\operatorname{Map}_{\bullet}^{a \sqcup *}(\mathcal{A}, K)$. Let $\operatorname{Map}_{\bullet}^{A, s}(\mathcal{A}, K) \subset \operatorname{Map}_{\bullet}^{A}(\mathcal{A}, K)$ consist of maps $\rho$ such that the subset $\rho^{-1}(K-*) \subset A$ either has one element or is the empty set. Consider a $\Gamma$-subspace $\Gamma_{A}^{\prime} \subset \Gamma_{A}$ such that $\Gamma_{A}^{\prime}(K, *)=$ $\operatorname{Map}_{\bullet}^{A, s}(\mathcal{A}, K)$.

The $\Gamma$-space $\Gamma_{A}$ is isomorphic to the $\Gamma$-space $\prod_{a \in(A-*)} \Gamma_{a}$. The $\Gamma$-space $\Gamma_{A}^{\prime}$ is isomorphic to the $\Gamma$-space $\vee_{a \in(A-*)} \Gamma_{a}^{\prime}$. Moreover these isomorphisms are consistent with the inclusion $\vee_{a \in(A-*)} \Gamma_{a} \subset \prod_{a \in(A-*)} \Gamma_{a}$.

Lemma B.2. Let $\mathcal{A}, I$ and $A(i)$ be as in Lemma B.1. There are two $\Gamma$-subspaces $\cup_{i \in I} \Gamma_{A(i)}^{\prime}, \cup_{i \in I} \Gamma_{A(i)}$ of the $\Gamma$-space $\Gamma_{\mathcal{A}}^{f}$. Suppose $\cup_{i \in I} A(i)=\mathcal{A}$. Then $\vee_{a \in(\mathcal{A}-*)} \Gamma_{a}=$ $\cup_{i \in I} \Gamma_{A(i)}^{\prime}$ and we have natural inclusions

$$
\vee_{a \in(\mathcal{A}-*)} \Gamma_{a}=\cup_{i \in I} \Gamma_{A(i)}^{\prime} \hookrightarrow \cup_{i \in I} \Gamma_{A(i)}
$$

of $\Gamma$-spaces.

Let $S e g: \Gamma-$ spaces $\rightarrow S^{1}-$ spectra be the functor associating the Segal $S^{1}$ spectrum to a $\Gamma$-space. Then $\operatorname{Seg}\left(\Gamma_{\mathcal{A}}^{f}\right)=\mathbb{S}_{\mathcal{A}}^{f}$. Given a non-distinguished element $a \in \mathcal{A}$ one has $\operatorname{Seg}\left(\Gamma_{a}\right)=\mathbb{S}$ and the functor $\operatorname{Seg}$ converts the inclusion $\Gamma_{a} \subset \Gamma_{\mathcal{A}}^{f}$ to the inclusion $\mathbb{S}_{a} \subset \mathbb{S}_{\mathcal{A}}^{f}$. For any finite pointed subset $A$ in $\mathcal{A}$, the functor $S e g$ converts the inclusion $\Gamma_{A} \subset \Gamma_{\mathcal{A}}^{f}$ to the inclusion $\mathbb{S}_{A} \subset \mathbb{S}_{\mathcal{A}}^{f}$. It also converts the inclusion $\Gamma_{A}^{\prime} \subset \Gamma_{A}$ to the inclusion $\mathbb{S}_{A}^{\prime} \subset \mathbb{S}_{A}$ as well as the inclusion $\vee_{a \in(\mathcal{A}-*)} \Gamma_{a} \subset$ $\Gamma_{\mathcal{A}}^{f}$ to the inclusion $\vee_{a \in(\mathcal{A}-*)} \mathbb{S}_{a} \subset \mathbb{S}_{\mathcal{A}}^{f}$.

The above arguments together with Lemmas B.1 and B.2 prove the following

Lemma B.3. Let $\mathcal{A}, I$ and $A(i)$ be as in Lemma B.1. There are two $S^{1}$-subspectra $\cup_{i \in I} \operatorname{Seg}\left(\Gamma_{A(i)}^{\prime}\right), \cup_{i \in I} \operatorname{Seg}\left(\Gamma_{A(i)}\right)$ of the $S^{1}$-spectrum $\operatorname{Seg}\left(\Gamma_{\mathcal{A}}^{f}\right)$. Suppose $\cup_{i \in I} A(i)=$ $\mathcal{A}$. Then $\vee_{a \in(\mathcal{A}-*)} \operatorname{Seg}\left(\Gamma_{a}\right)=\cup_{i \in I} \operatorname{Seg}\left(\Gamma_{A(i)}^{\prime}\right)$ and the inclusion

$$
\vee_{a \in(\mathcal{A}-*)} \operatorname{Seg}\left(\Gamma_{a}\right)=\cup_{i \in I} \operatorname{Seg}\left(\Gamma_{A(i)}^{\prime}\right) \hookrightarrow \cup_{i \in I} \operatorname{Seg}\left(\Gamma_{A(i)}\right)
$$

is a stable equivalence of $S^{1}$-spectra.

Notation B.4. Let $U, X \in S m / k$ and let $m, n \geqslant 0$ be integers. Set $\mathcal{A}=\mathrm{F}_{m}(U, X \times$ $\left.T^{n}\right)$ and regard it as a pointed set pointed by the empty framed correspondence $0_{m}$. Set $I=\operatorname{Fr}_{m}\left(U, X \times T^{n}\right)-0_{m}$.

In the remaining part of this section we use notation from Section 5.

Definition B.5. Given $\Phi=(Z, W, \varphi ; g), \Phi^{\prime}=\left(Z^{\prime}, W^{\prime}, \varphi^{\prime} ; g^{\prime}\right) \in \operatorname{Fr}_{m}\left(U, X \times T^{n}\right)$, we write $\Phi^{\prime} \leqslant \Phi$ if there is a closed subset $Z^{\prime \prime}$ in $\mathbb{A}^{m} \times U$ such that $Z=Z^{\prime} \sqcup Z^{\prime \prime}$ and

$$
\left(Z^{\prime}, W^{\prime}, \varphi^{\prime} ; g^{\prime}\right)=\left(Z^{\prime}, W-Z^{\prime \prime},\left.\varphi\right|_{W-Z^{\prime \prime}} ;\left.g\right|_{W-Z^{\prime \prime}}\right) \in \operatorname{Fr}_{m}\left(U, X \times T^{n}\right) .
$$

For any $\Phi \in \operatorname{Fr}_{m}\left(U, X \times T^{n}\right)$ set $A(\Phi)=\left\{\Psi \in \mathrm{F}_{m}\left(U, X \times T^{n}\right): \Psi \leqslant \Phi\right\} \subset \mathcal{A}$. Clearly,

$$
\mathcal{A}=\cup_{\Phi \in I} A(\Phi) \text {. }
$$

For a finite pointed set $(K, *)$ the pointed set $\operatorname{Fr}_{m}\left(U,\left(X \times T^{n}\right) \otimes K\right)$ is defined by the formula (8.1). Let $K^{\prime}=K-*$. By Definition 2.1(III) the set $\operatorname{Fr}_{m}(U,(X \times$ $\left.\left.T^{n}\right) \otimes K\right)$ consists of equivalence classes of tuples $(Z, W, \varphi ; g ; f)$, where $Z$ is a closed 
subset of $U \times \mathbb{A}^{m}$, finite over $U, W$ is an étale neighborhood of $Z$ in $U \times \mathbb{A}^{m}$, $\varphi_{1}, \ldots, \varphi_{m}, \varphi_{m+1}, \ldots, \varphi_{m+n}$ are regular functions on $W,(g, f): W \rightarrow X \times K^{\prime}$ is a regular map such that $Z=Z\left(\varphi_{1}, \ldots, \varphi_{m+n}\right)$. Notice that regular maps from $W$ to $X \otimes K$ are in one-to-one correspondence with couples of regular maps ( $W \rightarrow$ $\left.X, W \rightarrow K^{\prime}\right)$.

For a finite pointed set $(K, *)$, the pointed set $\mathrm{F}_{m}\left(U,\left(X \times T^{n}\right) \otimes K\right)$ is defined by the formula (8.2). By Definition 2.4 it consists of those elements $(Z, W, \varphi ; g ; f) \in$ $\operatorname{Fr}_{m}\left(U,\left(X \times T^{n}\right) \otimes K\right)$ such that the closed subset $Z$ of $U \times \mathbb{A}^{m}$ is connected.

Definition B.6. Denote by $\Gamma_{m}\left(U, X \times T^{n}\right)$ the $\Gamma$-space $(K, *) \mapsto \operatorname{Fr}_{m}(U,(X \times$ $\left.\left.T^{n}\right) \otimes K\right)$.

Similarly, $\Gamma_{m}^{\prime}\left(U, X \times T^{n}\right)$ stands for the $\Gamma$-space $(K, *) \mapsto \mathrm{F}_{m}\left(U,\left(X \times T^{n}\right) \otimes K\right)$.

Given $\Phi \in I$ define $\Gamma_{m}\left(U, X \times T^{n}\right)_{\Phi}$ as a $\Gamma$-subspace of the $\Gamma$-space $\Gamma_{m}\left(U, X \times T^{n}\right)$ such that for a finite pointed set $(K, *)$

$$
\begin{aligned}
& \Gamma_{m}\left(U, X \times T^{n}\right)_{\Phi}(K)= \\
& \left\{(Z, W, \varphi ; g ; f) \in \operatorname{Fr}_{m}\left(U,\left(X \times T^{n}\right) \otimes K\right) \mid(Z, W, \varphi ; g) \leqslant \Phi \in \operatorname{Fr}_{m}\left(U, X \times T^{n}\right)\right\} .
\end{aligned}
$$

Define $\Gamma_{m}^{\prime}\left(U, X \times T^{n}\right)_{\Phi}$ as a $\Gamma$-subspace of the $\Gamma$-space $\Gamma_{m}^{\prime}\left(U, X \times T^{n}\right)$ such that for a finite pointed set $(K, *)$

$$
\begin{aligned}
& \Gamma_{m}^{\prime}\left(U, X \times T^{n}\right)_{\Phi}(K)= \\
& \left\{(Z, W, \varphi ; g ; f) \in \mathrm{F}_{n}\left(U,\left(X \times T^{n}\right) \otimes K\right) \mid(Z, W, \varphi ; g) \leqslant \Phi \in \mathrm{F}_{m}\left(U, X \times T^{n}\right)\right\} .
\end{aligned}
$$

Definition B.7. For a finite pointed set $(K, *)$ put $K^{\prime}=K-*$ and consider a pointed set map

$$
\text { inc }_{K}: \operatorname{Fr}_{m}\left(U,\left(X \times T^{n}\right) \otimes K\right) \rightarrow \operatorname{Map}_{\text {Sets. }}^{f}(\mathcal{A}, K),
$$

which is defined as follows. Let $\Psi=(Z, W, \varphi ; g ; f) \in \operatorname{Fr}_{m}\left(U,\left(X \times T^{n}\right) \otimes K\right)$ and $a=\left(Z_{a}, W_{a}, \varphi_{a} ; g_{a}\right) \in \mathcal{A}=\mathrm{F}_{m}\left(U, X \times T^{n}\right)$. If the element $a$ is in $\mathcal{A}-A((Z, W \varphi ; g))$, then the map $\operatorname{inc}_{K}(\Psi)$ takes the element $a$ to the distinguished point $*$ of the set $K$. If $a \in A((Z, W, \varphi ; g))-0_{m}$, then the map $i n c_{K}(\Psi)$ takes the element $a$ to $f\left(Z_{a}\right) \in K^{\prime} \subset K$. Finally, the map $i n c_{K}(\Psi)$ sends $0_{m}$ to the distinguished point * of the set $K$.

Recall that $Z_{a}$ is connected and if $a \in A((Z, W, \varphi ; g))$, then $Z=Z_{a} \sqcup Z^{\prime \prime}$ for some $Z^{\prime \prime}$. Define a $\Gamma$-space morphism

$$
i n c_{m}: \Gamma_{m}\left(U, X \times T^{n}\right) \rightarrow \Gamma_{\mathcal{A}}^{f}
$$

sending a finite pointed set $(K, *)$ to the pointed set map $i n c_{K}$. It is straightforward to check that it is indeed a $\Gamma$-space morphism.

The following lemma is crucial.

Lemma B.8. The $\Gamma$-space morphism inc ${ }_{m}$ is injective. Moreover, using this inclusion the following identifications hold:

(1) for any $\Phi \in I$, one has $\Gamma_{m}\left(U, X \times T^{n}\right)_{\Phi}=\Gamma_{A(\Phi)}$ and $\cup_{\Phi \in I} \Gamma_{m}(U, X \times$ $\left.T^{n}\right)_{\Phi}=\cup_{\Phi \in I} \Gamma_{A(\Phi)}$

(2) for any $\Phi \in I$, one has $\Gamma_{m}^{\prime}\left(U, X \times T^{n}\right)_{\Phi}=\Gamma_{A(\Phi)}^{\prime}$ and $\cup_{\Phi \in I} \Gamma_{m}^{\prime}(U, X \times$ $\left.T^{n}\right)_{\Phi}=\cup_{\Phi \in I} \Gamma_{A(\Phi)}^{\prime}$

(3) for any $a \in \mathcal{A}-0_{m}=\mathrm{F}_{m}\left(U, X \times T^{n}\right)-0_{m}$, one has $\Gamma_{m}\left(U, X \times T^{n}\right)_{a}=\Gamma_{a}$;

(4) $\vee_{a \in(\mathcal{A}-*)} \Gamma_{m}\left(U, X \times T^{n}\right)_{a}=\vee_{a \in(\mathcal{A}-*)} \Gamma_{a}$. 
Applying the Segal functor Seg, we see that Lemmas B.2 and B.3 imply the following

Proposition B.9. Let $\mathcal{A}=\mathrm{F}_{m}\left(U, X \times T^{n}\right), I=\operatorname{Fr}_{m}\left(U, X \times T^{n}\right)-0_{m}$ be as Notation B.4 and for $\Phi \in I$ let the subset $A(\Phi) \subset \mathcal{A}$ be as in Definition B.5. There are two $S^{1}$-subspectra $\cup_{i \in I} \operatorname{Seg}\left(\Gamma_{m}^{\prime}\left(U, X \times T^{n}\right)_{\Phi}\right), \cup_{i \in I} \operatorname{Seg}\left(\Gamma_{m}\left(U, X \times T^{n}\right)_{\Phi}\right)$ of the $S^{1}$-spectrum $\operatorname{Seg}\left(\Gamma_{\mathcal{A}}^{f}\right)$. One has an equality of the $S^{1}$-subspectra

$$
\vee_{a \in(\mathcal{A}-*)} \operatorname{Seg}\left(\Gamma_{m}\left(U, X \times T^{n}\right)_{a}\right)=\cup_{\Phi \in I} \operatorname{Seg}\left(\Gamma_{m}^{\prime}\left(U, X \times T^{n}\right)_{\Phi}\right)
$$

and the inclusion

$$
\begin{aligned}
& \vee_{a \in(\mathcal{A}-*)} \operatorname{Seg}\left(\Gamma_{m}\left(U, X \times T^{n}\right)_{a}\right)=\cup_{\Phi \in I} \operatorname{Seg}\left(\Gamma_{m}^{\prime}\left(U, X \times T^{n}\right)_{\Phi}\right)= \\
& \operatorname{Seg}\left(\Gamma_{m}^{\prime}\left(U, X \times T^{n}\right)\right) \hookrightarrow \cup_{\Phi \in I} \operatorname{Seg}\left(\Gamma_{m}\left(U, X \times T^{n}\right)_{A(\Phi)}\right)=\operatorname{Seg}\left(\Gamma_{m}\left(U, X \times T^{n}\right)\right)
\end{aligned}
$$

is a stable equivalence of the $S^{1}$-spectra.

Set,

$\operatorname{Fr}_{m}^{S^{1}}\left(U, X \times T^{n}\right)=\operatorname{Seg}\left(\Gamma_{m}\left(U, X \times T^{n}\right)\right)$ and $\operatorname{Fr}_{m}^{S^{1}}\left(U, X \times T^{n}\right)_{\Phi}=\operatorname{Seg}\left(\Gamma_{m}\left(U, X \times T^{n}\right)_{\Phi}\right)$,

$\mathrm{F}_{m}^{S^{1}}\left(U, X \times T^{n}\right)=\operatorname{Seg}\left(\Gamma_{m}^{\prime}\left(U, X \times T^{n}\right)\right)$ and $\mathrm{F}_{m}^{S^{1}}\left(U, X \times T^{n}\right)_{\Phi}=\operatorname{Seg}\left(\Gamma_{m}^{\prime}\left(U, X \times T^{n}\right)_{\Phi}\right)$.

Under this notation the preceding proposition implies the following

Theorem B.10. Let $U, X \in S m / k$ and let $m, n \geqslant 0$ be integers. One has an equality of $S^{1}$-subspectra

$\vee_{\Psi \in\left(\mathrm{F}_{m}\left(U, X \times T^{n}\right)-0_{m}\right)} \mathrm{F}_{m}^{S^{1}}\left(U, X \times T^{n}\right)_{\Psi}=\cup_{\Phi \in\left(\operatorname{Fr}_{m}\left(U, X \times T^{n}\right)-0_{m}\right)} \mathrm{F}_{m}^{S^{1}}\left(U, X \times T^{n}\right)_{\Phi}$

of the spectra $\operatorname{Fr}_{m}^{S^{1}}\left(U, X \times T^{n}\right)$ and the inclusion

$$
\mathrm{F}_{m}^{S^{1}}\left(U, X \times T^{n}\right) \subset \operatorname{Fr}_{m}^{S^{1}}\left(U, X \times T^{n}\right)
$$

is a stable equivalence of the $S^{1}$-spectra.

We are now in a position to prove Theorem 1.2.

Proof of Theorem 1.2. This follows from Theorem B.10. Indeed, consider the composite morphism of $S^{1}$-spectra

$$
\mathbb{Z} \mathrm{F}_{m}^{S^{1}}\left(U, X \times T^{n}\right) \rightarrow \mathbb{Z}_{\mathrm{Fr}_{m}}^{S^{1}}\left(U, X \times T^{n}\right) \stackrel{\lambda_{X \times T^{n}}}{\longrightarrow} \mathbb{Z} \mathrm{F}_{m}^{S^{1}}\left(U, X \times T^{n}\right),
$$

where the left arrow is induced by the arrow (B.1). Within Definitions 2.4 and B.6, Theorem B.10 implies the left arrow is a stable equivalence of $S^{1}$-spectra. Note that the composite morphism is the identity map. Thus the morphism $\lambda_{X \times T^{n}}$ is a stable equivalence of $S^{1}$-spectra. This finishes the proof.

\section{REFERENCES}

[AGP] A. Ananyevskiy, G. Garkusha, I. Panin, Cancellation theorem for framed motives of algebraic varieties, preprint arXiv:1601.06642.

[BF] A. K. Bousfield, E. M. Friedlander, Homotopy theory of $\Gamma$-spaces, spectra, and bisimplicial sets, In Geometric applications of homotopy theory II, Lecture Notes in Math. 658, Springer Verlag, 1978, pp. 80-130.

[FP] R. Fedorov, I. Panin, A proof of Grothendieck-Serre conjecture on principal bundles over a semilocal regular ring containing an infinite field, Publ. Math. Inst. Hautes Etudes Sci. 122 (2015), 169-193.

[G] S. Greco, Henselization of a ring with respect to an ideal, Trans. Amer. Math. Soc. 144 (1969), 43-65. 
[GP1] G. Garkusha, I. Panin, Framed motives of algebraic varieties (after V. Voevodsky), J. Amer. Math. Soc. 34(1) (2021), 261-313.

[GP2] G. Garkusha, I. Panin, Homotopy invariant presheaves with framed transfers, Cambridge J. Math. 8(1) (2020), 1-94.

[L] M. Levine, A comparison of motivic and classical stable homotopy theories, J. Topology 7 (2014), 327-362.

[Mi] J. Milne. Étale cohomology, Princeton Mathematical Series 33, Princeton University Press, 1980

[Sch] S. Schwede, An untitled book project about symmetric spectra, available at www.math.uni-bonn.de/people/schwede/SymSpec-v3.pdf (version April 2012).

[Voe] V. Voevodsky, Notes on framed correspondences, unpublished, 2001. Also available at https://www.math.ias.edu/vladimir/publications

[Voe10] V. Voevodsky, Simplicial radditive functors, J. K-Theory 5 (2010), 201-244.

Department of Mathematics, Swansea University, Fabian Way, Swansea SA1 8EN, UK

Email address: g.garkusha@swansea.ac.uk

Department of Mathematics, University of Western Ontario, London, Ontario N6A 5B7, CANADA

Email address: aneshito@uwo.ca

St. Petersburg Branch of V. A. Steklov Mathematical Institute, Fontanka 27, 191023 St. Petersburg, Russia

Email address: paniniv@gmail.com 